Draft version November 14, 2018

Preprint typeset using $\mathrm{LATE}_{\mathrm{E}} \mathrm{X}$ style emulateapj v. 08/22/09

\title{
EVOLVED STARS IN THE CORE OF THE MASSIVE GLOBULAR CLUSTER NGC $2419^{1}$
}

\author{
ERIC L. SANDquist, Jordan M. Hess \\ Department of Astronomy, San Diego State University, 5500 Campanile Drive, San Diego, CA 92182 \\ Draft version November 14, 2018
}

\begin{abstract}
We present an analysis of optical and ultraviolet Hubble Space Telescope photometry for evolved stars in the core of the distant massive globular cluster NGC 2419. We characterize the horizontal branch (HB) population in detail including corrections for incompleteness on the long blue tail. The majority of the horizontal branch stars can be identified with two main groups (one slightly bluer than the instability strip, and the other at the extreme end of the HB). We present a method for removing (to first order) lifetime effects from the distribution of HB stars to facilitate more accurate measurements of helium abundance for clusters with blue HBs and to clarify the distribution of stars reaching the zero-age HB. The population ratio $R=N_{H B} / N_{R G B}$ implies there may be slight helium enrichment among the EHB stars in the cluster, but that it is likely to be small $(\Delta Y<0.05)$. An examination of the upper main sequence does not reveal any sign of multiple populations indicative of helium enrichment.

The stellar distribution allows us to follow how the two main types of stars evolve after the HB. We find that the transition from stars that reach the asymptotic giant branch to stars that remain at high temperatures probably occurs among the extreme horizontal branch stars (EHB) at a larger temperature than predicted by canonical evolution models, but qualitatively consistent with heliumenriched models. Through comparisons of optical CMDs, we present evidence that the EHB clump in NGC 2419 contains the end of the canonical horizontal branch, and that the boundary between the normal HB stars and blue hook stars shows up as a change in the density of stars in the CMD. This corresponds to a spectroscopically-verified gap in NGC 2808 and an "edge" in $\omega$ Cen. The more clearly visible HB gap at $V \sim 23.5$ identified by Ripepi et al. (2007) appears to be too bright.

Once corrected for lifetime effects, we find that NGC 2419 is currently converting about $25-31 \%$ of the first-ascent red giant stars in its core into extreme blue horizontal branch stars - the largest fraction for any known globular cluster. A comparison of upper red giant branch with theoretical models indicates there is a slight deficiency of bright red giant stars. This deficiency occurs far enough below the tip of the red giant branch that it is unlikely to be associated with the production of extreme horizontal branch stars via strong mass loss before the core helium flash.

Subject headings: stars: horizontal branch — stars: evolution — stars: luminosity function — stars: mass loss — globular clusters: individual (NGC 2419)
\end{abstract}

\section{INTRODUCTION}

NGC 2419 is the fourth brightest globular cluster known in the Milky Way $\left(M_{V}=-9.58\right.$; Harris 1996). NGC 2419's large mass would normally make it ideal for studying short phases in the lives of evolved low-mass stars because massive cluster (having more stars) should produce a greater chance of catching some stars in those phases. Until recently though, it has been neglected because it resides far in the outer halo of the Milky Way (approximately $90 \mathrm{kpc}$ from the center). Massive clusters can make it possible to observe stars in short-lived evolution phases because there is a greater chance of catching some star in such a phase when there are more chances.

Evolved stars have unusually strong influences on the luminosity-integrated properties of a galaxy: in old stellar populations, the overall color and the ultraviolet emission result from them. The luminous giants at the tip of the red giant branch (TRGB) are frequently used as stan-

\footnotetext{
Electronic address: erics@sciences.sdsu.edu

${ }^{1}$ Based on observations with the NASA/ESA Hubble Space Telescope, obtained at the Space Telescope Science Institute, which is operated by the Association of Universities for Research in Astronomy, Inc., under NASA Contract NAS 5-26555.
}

dard candles in resolved stellar populations. Extremely blue HB (hereafter, EHB) stars are a leading candidate for galactic ultraviolet emission. These populations are linked at least in an evolutionary sense, but it remains a longstanding problem to explain the details of how $\mathrm{HB}$ stars (and more specifically the EHB stars) are produced. There has been much recent interest in multiple stellar populations having different chemical compositions (particularly helium, but also heavier elements) within individual clusters (NGC 2808, D'Antona et al. 2005; $\omega$ Cen, Bedin et al. 2004; M13, Caloi \& D'Antona 2005; NGC 6218, Carretta et al. 2007; NGC 6388, Busso et al. 2007; NGC 6441, Caloi \& D'Antona 2007) However, even allowing for composition effects, it still seems to be necessary for giant stars to lose most of the mass in their envelopes if EHB stars are to be produced - the turnoff mass of a helium-enriched population cannot be reduced enough to have a giant star consume its envelope before it reaches the TRGB.

In this paper, we examine deep photometry of the evolved stars in the core of the cluster using high resolution Hubble Space Telescope (HST) imagery. We have three main goals: to characterize brief evolutionary phases and their effects on the integrated light of the clus- 
ter, to examine the paths (often brief) that lead from one evolutionary phase to another, and to use the stellar populations to constrain chemical evolution within the cluster. EHB and bright red giant branch (RGB) stars are particularly important to the integrated colors of clusters. EHB stars can be seen in several earlier CMDs for NGC 2419 (Harris 1997; Stetson 2005), although the impression was that the population was small due to incompleteness at the faint end. Dalessandro et al. (2008) recently presented a reduction of a portion of the available archived $H S T$ data for the cluster that clearly shows the faint end of the blue HB tail, and that the EHB population is a substantial fraction of all $\mathrm{HB}$ stars in the cluster. In section 3.1, we conduct a deeper analysis of the HB population of the cluster.

The details of the transition from the RGB to the HB remain poorly understood. In the case of the massive cluster NGC 2808, Sandquist \& Martel (2007) found a deficit of bright red giants compared to theoretical predictions that may be linked to the EHB stars there - if a star loses enough mass on the red giant, it can leave the red giant branch before the helium flash at the TRGB, igniting helium later in a "hot flash" that ultimately deposits it at the blue end of the HB. NGC 2419 appears to be more efficient at producing EHB stars than most clusters, so in 3.3 we examine the upper RGB for signatures of EHB star production.

Integrated luminosity has been linked to a number of unusual groups of stars within globular clusters, including blue stragglers (their relative frequency is anticorrelated with total luminosity), extremely blue horizontal branch stars, and populations with different age and/or chemical composition. To date, NGC 2419 has shown little indication that it contains anything but a stellar population with a single composition $([\mathrm{Fe} / \mathrm{H}]$ $=-2.1$; Suntzeff et al. 1988) and age. However, NGC 2419 is now known to have a strongly double-peaked HB, and so we examine the helium abundance indicator $R$ in \3.5.

\section{OBSERVATIONS AND DATA REDUCTION}

This study has focused on the evolved stellar populations of the cluster core. The archival HST datasets that were reduced are listed in Table 1, and a map of the fields is shown in Fig. 1. Images from the ACS WFC and HRC instruments were processed using the DOLPHOT photometry package ${ }^{2}$ with its module tuned for ACS data. Individual frames were obtained from the HST archive in order to get photometry on the giant stars. The WFPC2 images were analyzed using the HSTPhot ${ }^{3}$ photometry package (Dolphin 2000). Although different filters were used for imaging in different epochs, the fields overlapped in many cases, and in each field we have photometry in the F555W and F814W filters.

An RA-DEC offset coordinate system was set up using the WFPC2 datasets. Positions are given relative to the cluster center given by Harris (1996). This position agrees extremely well with the determination of the center of gravity by Dalessandro et al. (2008). Images in the ACS system are subject to a lot of geometric distortion due to the off-axis positions of the cameras,

\footnotetext{
2 http://purcell.as.arizona.edu/ andy/dolphot/
}

3 http://purcell.as.arizona.edu/hstphot/

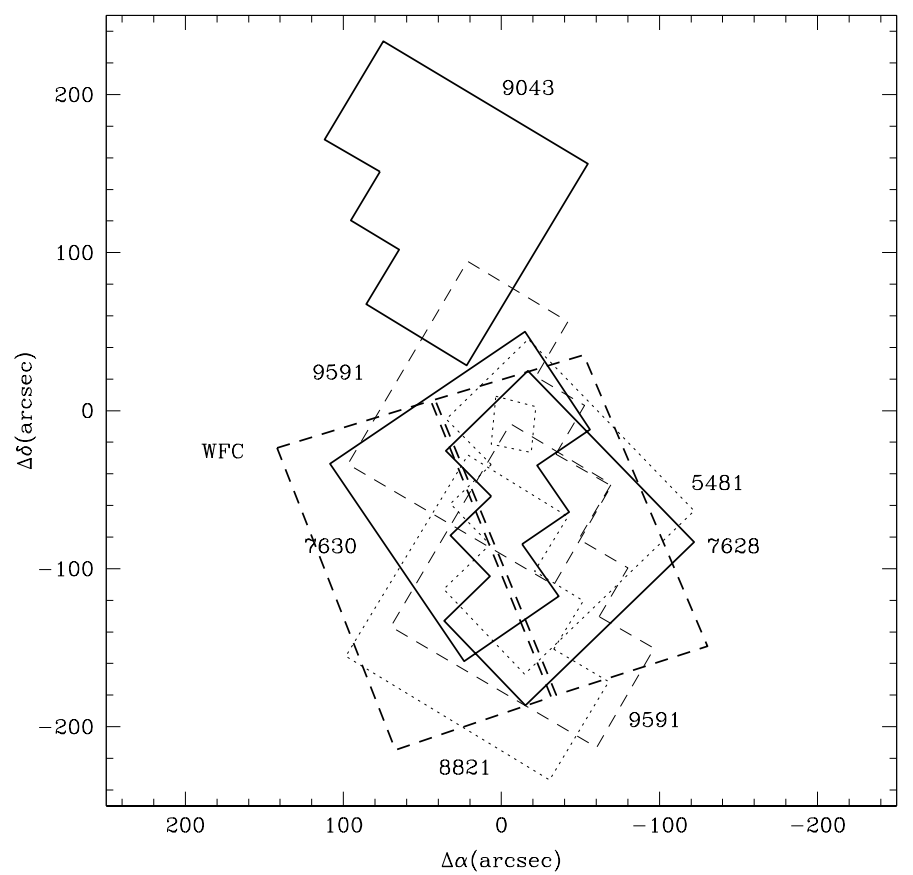

FIG. 1.- Sky positions of HST fields that were analyzed in this paper. Positions are given in arcseconds relative to the Harris (1996) position given for the cluster center. WFPC2 fields are identified with the proposal ID number (see Table 1) except where the WFPC2 field almost completely overlaps another WFPC2 field. The deepest photometry was taken in the fields having bold lines. The ACS HRC field is the small rectangle surrounding $(0,0)$.

so we used fourth-order geometric transformations obtained from the most recent Image Distortion Correction Table (IDCTAB) file (qbu1641sj_idc.fits) ${ }^{4}$ in order to convert pixel positions into relative sky positions. Coordinates for stars in the WFPC2 images were derived from the METRIC task in the IRAF package STSDAS. METRIC positions typically have absolute position errors that are a significant fraction of an arcsecond, but we are primarily interested in relative positions. After initial identifications of stars were made in the different datasets, preliminary coordinate transformations were derived for all of the WFPC2 and ACS fields. The coordinate transformations were then refined with additional cross-identifications, and these were used to match up all stars measured in the datasets.

The HST datasets were analyzed to maximize the detection of different populations of evolved stars in the cluster. Identification of horizontal branch stars is generally more effective in shorter wavelength filters when most of the HB stars are bluer than the RR Lyrae instability strip, as in NGC 2419. In this case, however, the optical CMDs from the ACS WFC field were deep and had high spatial resolution. At the same time, the optical CMDs were significantly incomplete in the core of the cluster even with the high resolution. In Fig. 2, we show the optical WFC CMD for samples in and outside $60^{\prime \prime}$ from the cluster center. From artificial star tests (see \$2.1), we find that the WFC data is more than $80 \%$ com-

\footnotetext{
${ }^{4}$ http://www.stsci.edu/hst/observatory/cdbs/SIfileInfo/ACS/ACSDistortion
} 
TABLE 1

HST DATASETS FOR NGC 2419

\begin{tabular}{clcl}
\hline \hline Prop. ID $^{\text {a }}$ & \multicolumn{1}{c}{ P.I. } & Instrument & \multicolumn{1}{c}{ Filters } \\
\hline 5481 & Hesser & WFPC2 & F555W, F814W \\
\hline 7628 & Whitmore & WFPC2 & F336W, F380W, F439W, F450W, F555W, F675W, F814W \\
8095 & Ibata & WFPC2 & F555W \\
9601 & Koekemoer & WFPC2 & F300W, F336W, F439W, F450W, F675W \\
\hline 7630 & Casertano & WFPC2 & F300W, F555W, F814W \\
6937 & Stiavelli & WFPC2 & F555W \\
\hline 8821 & Riess & WFPC2 & F555W, F814W \\
\hline 9043 & Tonry & WFPC2 & F555W, F814W \\
\hline 9591 & Whitmore & WFPC2 & F814W \\
\hline 9666 & Gilliland & ACS WFC & F435W, F555W, F814W \\
\hline 10815 & Brown & ACS HRC & F250W \\
\hline
\end{tabular}

a Observations with almost completely overlapping fields are grouped together.

plete even down to the faint end of the EHB for $r>60^{\prime \prime}$. Because they also covered the widest area, they became our primary choice for selection. Had the ultraviolet images been deeper, they would have been more reliable for detecting the bluest HB stars. The ACS HRC frames in the F250W filter covered a small field centered about $12^{\prime \prime}$ from the cluster core, allowing us to confidently identify 11 faint HB stars that would otherwise have been rejected by quality cuts that were applied to the next highest resolution dataset (the ACS WFC images). Fig. 3 shows CMDs of HB stars detected in both the HRC and WFC images. Based on these considerations, we believe the $\mathrm{HB}$ star sample in the HRC field is virtually $100 \%$ complete. Observations in the F336W filter of the proposal 7628 field also provide good detections of bright EHB stars, although the detection limit brighter than the faint end of the EHB (see Fig. 18). The situation in the F300W filter was similar, so they were only used for verification of brighter candidates.

Because some of the datasets have image sequences that are suitable for searching for variables, we checked the RR Lyrae population in the HST fields. We computed the $J$ statistic (Stetson 1996) for the combination of F555W and F814W images from the proposal ID 6937 and 7630 field, and found that this efficiently identified variable stars in the field. We confirmed most of the Pinto \& Rosino (1977) variables that fall in the field (V2, V3, V4, V6, V11, V13, V26, V29, V30, V33, V35, V38, V39, V40) and have detected others. The coordinates given for V37 were inconsistent with the Pinto \& Rosino finding chart position, which puts it outside the HST fields. The coordinates for V38 and V40 were both significantly in error, but the stars were identified using the finding chart and their variability was confirmed. We also were unable to find an obvious counterpart to their V41. Most of the RR Lyrae stars could be tentatively identified in the WFC datasets using their CMD positions, but there were 4 stars that ended up in the middle of the RGB as a result of the sampling of the light curve with a small number of observations.

The CMDs used for the selection of RGB stars are shown in Fig. 4. In the RGB star sample, the primary contaminant is AGB stars. For this reason we also relied heavily on CMDs with short wavelength filters in order to separate AGB and RGB stars by temperature. CMDs with ultraviolet observations on the magnitude axis can help turn temperature (color) differences into luminosity (magnitude) differences, which are often eas- ier to detect. Our primary choice for RGB identification used the F336W filter from the proposal 7628 field because this most cleanly and reliably separated AGB stars. As our second choice we used ACS WFC observations in the F435W filter and WFPC2 observations in the F439W and F450W filters. Stars in the longest WFC exposures were unfortunately saturated, although we were able to use saturated star photometry from DOLPHOT in most cases. (We have not included saturated star photometry in the averaged photometry described later.) If there was some ambiguity in identifying a star as RGB or AGB, it usually came in the CMDs due to (relatively) small color separation between RGB and AGB. The AGB clump can easily be seen in $19.9<F 435 W<20.5$, but the AGB sequence mostly merges with the RGB brighter than that. Photometry in the F300W (WFPC2) and F250W (HRC) filters were also useful, but again, we found that they were sometimes unreliable toward the tip of the giant branch due to measurement uncertainties. Toward the faint end of the sample though, there is a large and useful magnitude difference between the RGB and the AGB clump.

\subsection{Artificial Star Tests}

In order to fully characterize the faint HB population, we conducted a series of artificial star tests on the ACS WFC frames, which comprises the great majority of the surveyed field. Before we ran the tests, we first defined a fiducial line running through the blue HB tail using the $\mathrm{F} 435 \mathrm{~W}$ filter for the magnitude axis, and using both $F 435 W-F 555 W$ and $F 435 W-F 814 W$ colors. To ensure that the two artificial star colors were consistent, we used the photometry for a select group of stars to define the fiducial. Artificial star magnitudes were selected randomly with a flat luminosity function, and colors were generated from quadratic interpolation between the fiducial points.

In each of the artificial HB star tests, stars were placed in a grid of cells 30 pixels wide. The stars were randomly placed within cells, and the grid itself was shifted from test to test. A total of 5 runs containing 91926 stars were completed. Stars were identified as recovered if they were detected in the $\mathrm{F} 435 \mathrm{~W}$ and $\mathrm{F} 814 \mathrm{~W}$ images and passed cuts on sharpness $(|\mathrm{SHARP}|<0.5)$, $\chi^{2}(<5)$, and crowding. With regard to crowding, the photometry for a star is often affected by light from nearby neighbors. Photometry was derived from profile fitting in which overlapping stellar images are fit si- 


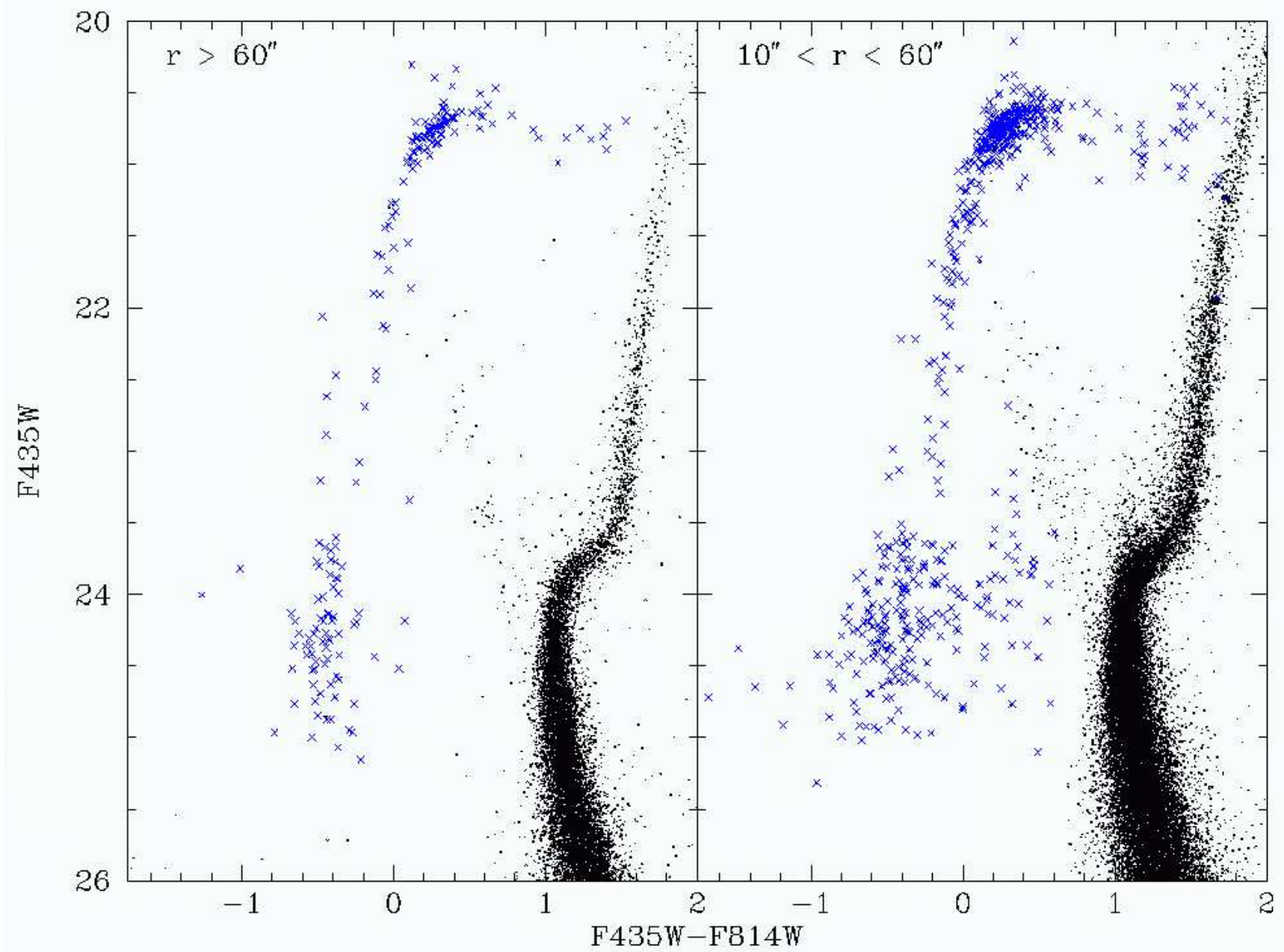

FIG. 2.- ACS WFC color-magnitude diagrams with horizontal branch stars passing criteria for detection in our artificial star trials (blue crosses; see $\$ 2.1$, divided by projected distance from the cluster center.

multaneously with point-spread functions. The crowding measure involves the resulting difference in brightness if nearby neighbors were not simultaneously fitted. Stars were rejected if they would have been more than $0.3 \mathrm{mag}$ brighter if nearby neighbors had been neglected. (While this probably rejected some real stars, if we apply the cuts consistently to the artificial stars and the real stars, we can derive accurate incompleteness corrections.)

For the analysis, stars were divided into annuli $\left(20^{\prime \prime}\right.$ wide; approximately one core radius) and magnitude (0.2 mag wide) bins. According to the artificial star tests (see Fig. 5), the bright HB sample is almost $100 \%$ complete throughout the HST fields. The worst incompleteness occurs in the cluster core, where EHB stars in the WFC field are only about $29 \%$ complete within one core radius. However, we used UV observations to improve the recovery of EHB stars in the densest part of the core. Because crowding is negligible in the HRC images and the faint end of the HB is well above the faint limit of the images, we have assumed that our HB sample is $100 \%$ complete in the HRC field.

\subsection{Photometry}

With such a heterogeneous set of images and filters, we spent some effort to merge the photometric datasets together where possible. We emphasize that the merged photometry was not used in categorizing stars by evolutionary state - that was done using individual datasets because the relative precision was higher. We used the observations of the WFPC2 proposal 7630 field as the reference because it showed the greatest amount of overlap with the other fields. (We found significant zeropoint differences between the two ACS WFC chips, which is why they were not used.) The photometry that could be merged in this way used the F300W, F555W, and F814W filters.

In the F555W filter, we found no signs of color dependence among the observations in the different datasets, so we only corrected for zeropoint differences. The offsets (labeled $c_{0}$ ) are provided in Table 2 and are in the sense of (7630 - dataset). Residuals versus F555W magnitude are plotted in Fig. 6. In the F814W filter, we found a significant color dependence in the residuals between the 5481 and 7630 photometry. Note that all of the WFPC2 photometry was rereduced using the same software and photometry parameters, so this difference appears to come from instrumental effects. Colordependent terms are labeled $c_{1}$ in the table. Residuals versus F814W magnitude are plotted in Fig. [7 and the dependence on color is shown in Fig. 8. We note that the color dependence of the transformation from the WFC to the WFPC2 in the F555W and F814W filters evalu- 

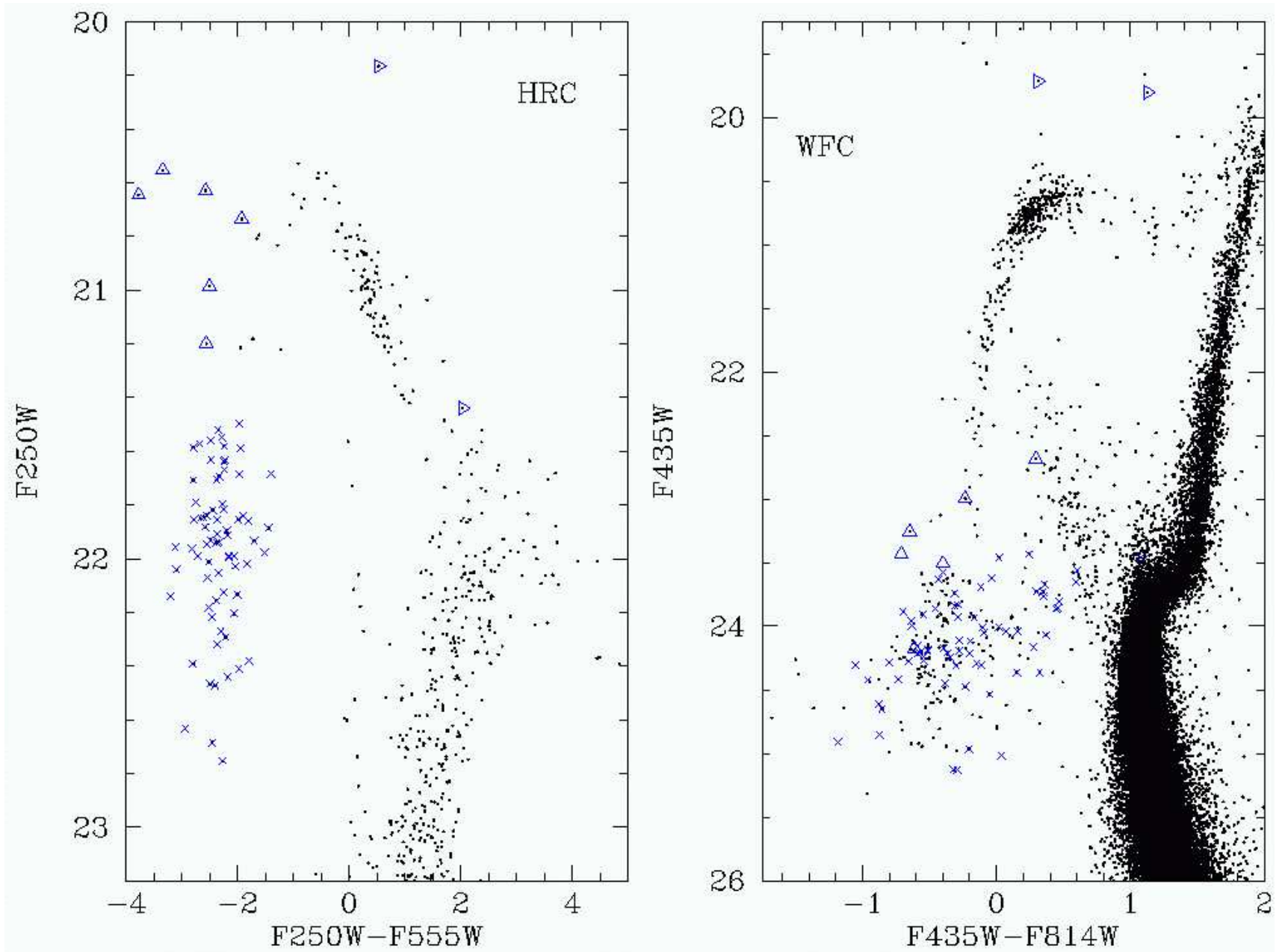

FIG. 3. - Color-magnitude diagrams showing the selection of extreme HB stars (blue crosses) from the ACS HRC F250W filter, and the corresponding stars in the ACS WFC field. Triangles show probable evolved HB stars in the ACS HRC field, with the triangles pointing roughly in the direction of their expected evolution (see 3.2 ).

TABLE 2

Photometric Transformation to Proposal 7630 РнотOMETRY

\begin{tabular}{|c|c|c|c|c|c|}
\hline \multirow{2}{*}{ Prop. ID } & \multicolumn{2}{|c|}{ F300W } & \multirow{2}{*}{$\begin{array}{c}\mathrm{F} 555 \mathrm{~W} \\
c_{0}\end{array}$} & \multicolumn{2}{|c|}{$\mathrm{F} 814 \mathrm{~W}$} \\
\hline & $c_{0}$ & $c_{1}$ & & $c_{0}$ & $c_{1}$ \\
\hline 5481 & & & -0.068 & -0.141 & 0.0778 \\
\hline 7628 & 0.113 & 0.0201 & -0.017 & -0.019 & \\
\hline 8821 & & & -0.005 & 0.004 & \\
\hline $9591(\mathrm{~S})^{\mathrm{a}}$ & & & & -0.005 & \\
\hline $9591(\mathrm{~N})^{\mathrm{a}}$ & & & & -0.032 & \\
\hline 10815 (WFC1-E) $^{\mathrm{b}}$ & & & -0.033 & 0.011 & \\
\hline $10815(\mathrm{WFC} 2-\mathrm{W})^{\mathrm{b}}$ & & & -0.078 & -0.037 & \\
\hline
\end{tabular}

a The two proposal ID 9591 fields are labeled south or north based on their positions, and both are shown in Fig. 1.

b The fields of the two ACS WFC chip fields in proposal ID 10815 fields are labeled east or west based on their positions, and are shown in Fig. 1

ated by Sirianni et al. (2005) are consistent with zero (to within $1 \sigma$ ) for all color choices.

We found a fairly large zeropoint difference and color dependence of the photometric residuals in the F300W filter. Time-dependent zeropoint changes are well-known in ultraviolet filters (Holtzman et al. 1995), but the behavior in F300W has not been thoroughly mapped out because the filter is infrequently used. We have therefore only corrected for relative differences between the two F300W datasets, again using the proposal 7630 photometry as the standard. (Of all of the filters considered here, the ultraviolet filters are most likely to have significant zeropoint differences in comparison to the flight photometric standard system.) The difference in F300W magnitudes as a function of magnitude and color are shown in Fig. 9.

The WFC F435W and WFPC2 F439W filters are also close in wavelength coverage, although the NGC 2419 field was only observed in these filters using WFPC2 in proposal 7628 . We compared the photometry, and found a small color term is able to do a good job of transforming the two. The color term $\left(c_{0}=0.030\right)$ is slightly larger than the one given in Table 20 of Sirianni et al. (2005), but does a better job at simultaneously bringing the HB and RGB populations into consistency. Fig. 10 shows the comparison between the WFPC2 and transformed WFC data.

We have generally left our photometry in the VEGAMAG flight system. When transformations to $U B V R I$ bandpasses are needed for comparisons to external photometry for other clusters, we have used transformation equations from Sirianni et al. (2005) for ACS WFC im- 

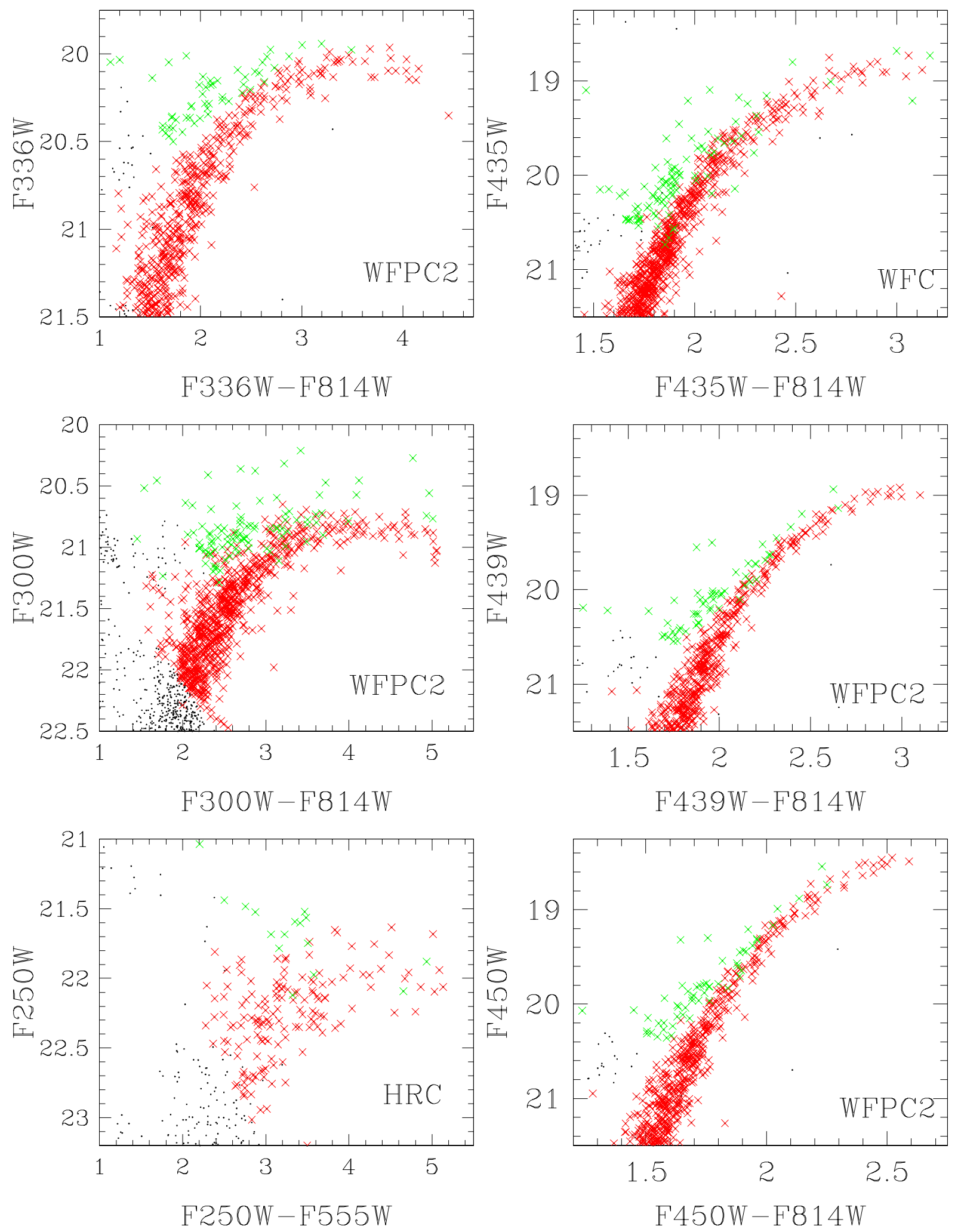

FIG. 4.- The color-magnitude diagrams used to select red giant branch branch (red) and asymptotic giant branch (green) stars in the HST fields. No cuts on position with the cluster were applied to any of the CMDs. 


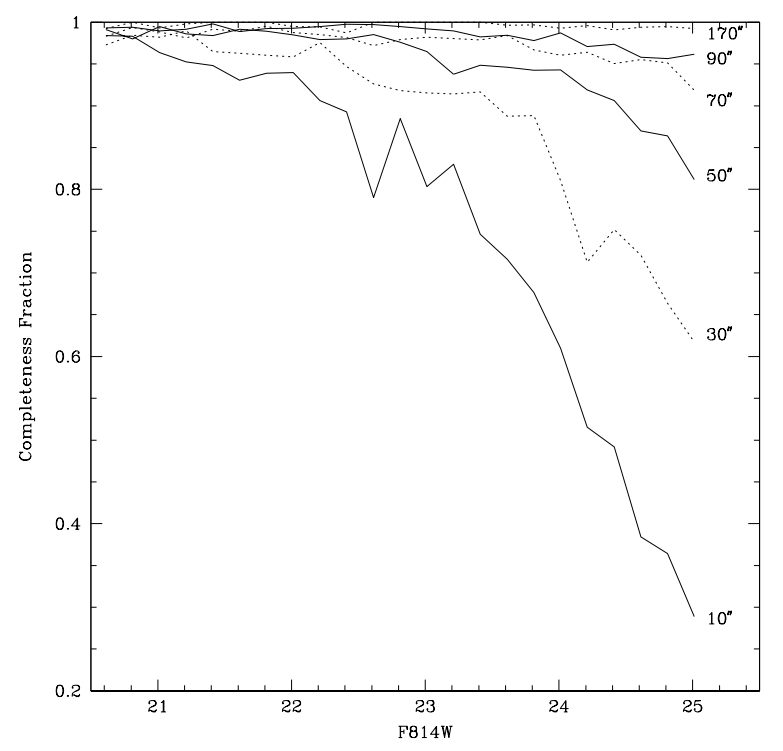

FIG. 5.- Completeness measures for artificial horizontal branch stars as a function of $\mathrm{F} 814 \mathrm{~W}$ magnitude and distance from the cluster center. The lines are identified with the radius of the center of the annulus (all annuli are $20^{\prime \prime}$ wide).

ages, and the most recent transformations maintained by A. Dolphin ${ }^{5}$ for WFPC2 images. The flight system photometry is presented in Table 4 .

\section{ANALYSIS}

\subsection{The Horizontal Branch}

The HB morphology has been recently described (based solely on ACS photometry) in Dalessandro et al. (2008), but the focus of that study was on the blue stragglers. Here we attempt to fully characterize the evolved populations, and in that vein we have identified $994 \mathrm{HB}$ stars in the union of the HST fields. Our $F 435 W$ and $F 814 W$ magnitudes from the ACS data are approximately equivalent to the $B$ and $I$ magnitudes from Dalessandro et al. . For most of the discussion below, the two pairs can be used almost interchangeably (color transformation coefficients are small; Sirianni et al. 2005). There are a number of interesting features of the CMDs deserving of comment.

The HB has an extremely large total extent in effective temperature, although the red end of the $\mathrm{HB}$ and the RR Lyrae instability strip are sparsely populated, as is theoretically expected for the cluster's metallicity. There are at least four clear breaks in the distribution of stars that can be seen in Fig. 11]

1. $B-I=0.7$ : This break approximately corresponds to the blue end of the instability strip, but there is a clear drop off in the number of stars redder than this point. Although we have been able to identify RR Lyrae variable stars from the datasets, we generally do not have good enough coverage of the light curve to derive precise average magnitudes.

2. $B \approx 21$ ): while not a gap in the distribution, this marks the blue edge of the most heavily populated

\footnotetext{
5 http://purcell.as.arizona.edu/wfpc2_calib/
}

part of the HB. The HB is more sparsely populated out to the next fainter break.

3. $B \approx I \approx 22.25$ : This is the brighter of the two clear gaps in the HB distribution. We will refer to the stars with $22.25<B<23.5$ as the inter-gap population.

4. $B \approx 23.5$ : The fainter of the two gaps marks the bright edge of the collection of extreme $\mathrm{HB}$ (EHB) and blue hook (BHk) stars identified by Dalessandro et al.. Those authors tentatively identified this as a gap.

Dalessandro et al. compared the HB distribution with the more massive cluster $\omega$ Cen, and found similarities in the overall distribution - notably comparable percentages of stars in the EHB/BHk group. The comparison with $(B, B-I)$ data for M13 in Fig. 11 clearly shows that NGC 2419's HB extends approximately 1 mag fainter than the greater part of the EHB clump for M13 (although a handful of stars have been identified fainter than the clump). A comparison with two other clusters with long blue HB tails (NGC 2808 and M15) is shown in Fig. 12. In each case, the number of HB stars appears to sharply drop at approximately the same magnitude (relative to the edge of the RR Lyrae instability strip): $B \sim 21$ in NGC 2808 , and $B \sim 25.1$ in NGC 2419. M15 is a cluster of similar $[\mathrm{Fe} / \mathrm{H}]$ and comparable but lower mass that converts only a has a tiny proportion of its stars into EHB stars. NGC 2808 has a mass that is considerably closer to that of NGC 2419, but it has a much higher $[\mathrm{Fe} / \mathrm{H}]$. It produces more EHB stars than M15, but the EHB stars are still a small fraction of the total HB stars.

The majority of the cluster's HB stars fall in a compact group in the CMD $(20.6<B<21,0.2<B-I<0.6$; see Figure 11), with less dense extensions to the red and blue. There is a fair amount of evidence to suggest that all or nearly all of the stars redward of the main peak have evolved to their present colors from the main peak. The presence of AGB stars (especially ones in a "clump" at the base of the AGB) implies redward evolution after the HB phase, and at least some models (see the next paragraph) predict stars should spend a substantial time redward of their starting point on the HB. Probably the strongest evidence comes from the comparison of the period-amplitude diagram for NGC 2419 RR Lyrae stars with that of M3 (Ripepi et al. 2007). M3 is a cluster which has strong populations of stars both redder and bluer than the instability strip, and so it is legitimate to expect from theoretical evolutionary tracks that there are relatively unevolved RR Lyrae stars as well as stars that have evolved into the instability strip from the blue HB. The lower average densities of the evolved stars are expected to result in larger periods, and this is supported by the appearance of two sequences in the periodamplitude diagram (Cacciari et al. 2005). NGC 2419 RR Lyraes fall nearly exclusively among the "evolved" population of M3 stars (see Fig. 3 of Ripepi et al. 2007). In this context, star V2 appears to be an unusually bright evolved RR Lyrae star. The CMD position identifies it as a "supra-HB" star (see \$3.2). We have identified V2 from the finding chart published by Pinto \& Rosino (1977), and from our own detection of its variability in the $H S T$ 


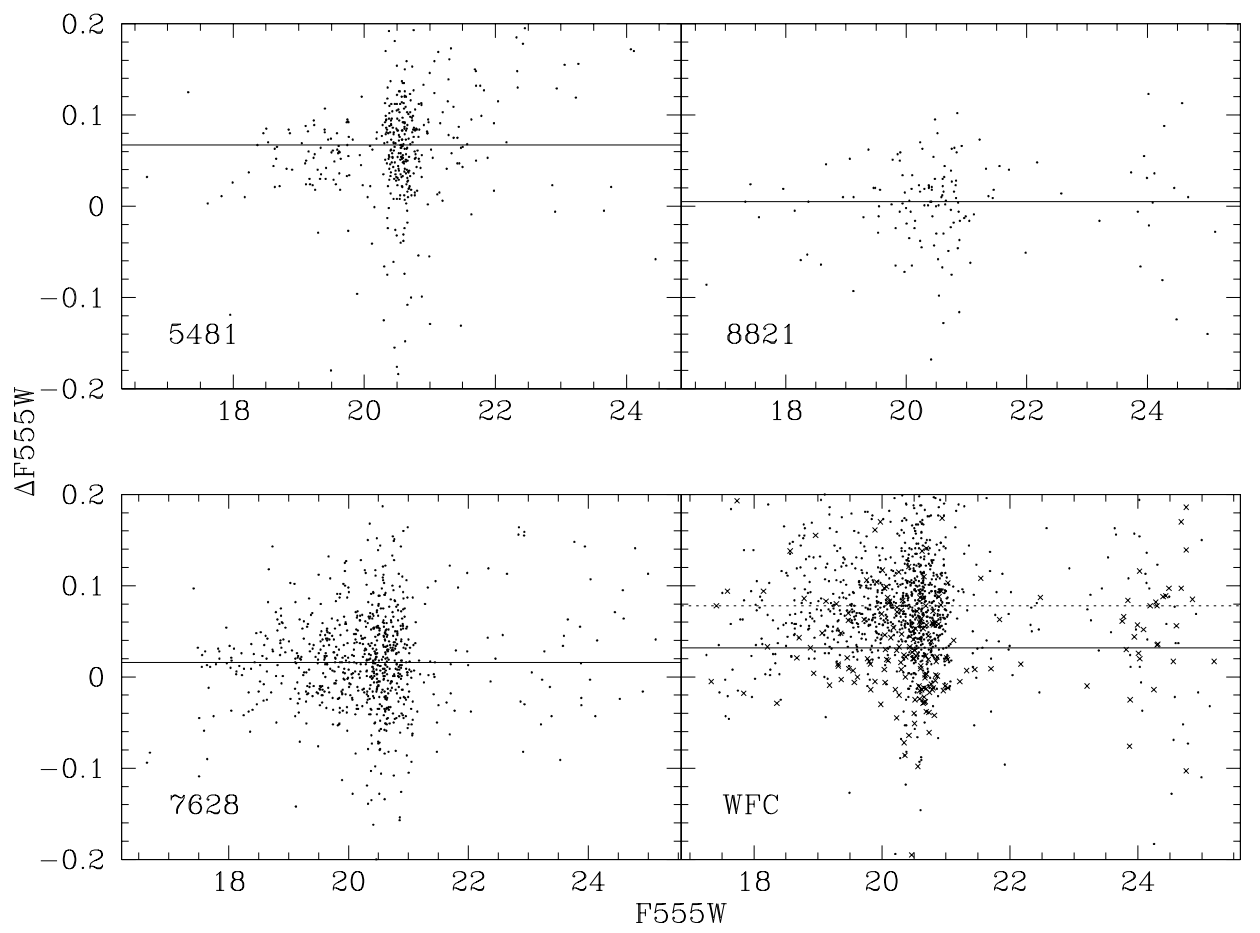

FIG. 6. - Residuals between F555W instrumental magnitudes in the sense of (7630 field - other). Lines show the median residuals. For the ACS WFC photometry, the solid line and $\times$ symbols are from the WFC2 chip (image extension 1 ), and dotted line and $\bullet$ symbols relate to the WFC1 chip (image extension 2).

data. Our photometry implies that it was observed near minimum light in the F814W filter (see Fig. 13). The only published light curve (Pinto \& Rosino 1977) implies it has a large amplitude (about $1.3 B$ magnitudes) and period, although their photometry indicates that it is comparable to other known RR Lyrae stars oscillating in the fundamental mode. At the same time, their photometry implies that it is also similar in brightness to other RR Lyraes as well. This contradiction between the Pinto \& Rosino photometry and ours suggests that this star should be studied in more depth.

HB sequences (e.g. Dotter et al. 2007) imply that zeroage horizontal branch (ZAHB) stars for NGC 2419's low metallicity and primordial helium abundance always fall bluer than the instability strip, even for stars with no mass loss. Synthetic HB models also predict a significant number of variable and red HB stars for a zero-age population that begins at the position of the main peak (see Fig. 20). Blueward evolution in HB stars tends to occur in stars with lower initial $L_{H e} / L$ (Iben \& Rood 1970), where $L_{H e}$ is the luminosity released by helium fusion. This means that the hydrogen-fusion shell rapidly adds mass to the helium core of the star. This can result from higher helium abundances or metallicities (both affecting the structure of the envelope, and producing higher shell fusion temperatures), but in general it requires a substantial hydrogen envelope in order to maintain the conditions needed for a strong hydrogenfusion shell (Sweigart 1987). When the hydrogen shell is weakened (by low envelope mass or low envelope helium abundance, for example), the color evolution is driven by the depletion of helium in the core as the star becomes more giant-like. So the evolution of HB stars can bear on questions of helium abundance, at least tangentially. The relatively low dispersion in magnitude among the red $\mathrm{HB}$ stars is consistent with the idea that the stars in the main peak have low (approximately primordial) helium abundance. An increase in $Y$ of as little as 0.05 produces an initial blue loop in the star's evolution, and the subsequent evolution toward the giant branch takes place at a significantly greater luminosity. (See Figure 9 of Busso et al. 2007 for a comparison of much more metal-rich tracks with different helium abundances.)

The more important issue is what can be learned about the population of the second peak on the EHB, as it is the bluer population of HB stars that is identified with helium-enriched gas when multiple populations are present. An understanding of this group is complicated by poorer sensitivity to temperature of the colors available here, and the fact that some of these stars appears to exist beyond the end of the canonical HB, as seen in Fig. 11. Because these stars are at or beyond the end of the $\mathrm{HB}$, they are called extreme $\mathrm{HB}$ or blue hook stars (we will refer to them as the $\mathrm{EHB} / \mathrm{BHk}$ group). There are major uncertainties in models of these stars: for example, it is still unclear exactly how the stars are formed and whether the formation mechanism has a strong effect on the position in the CMD, but the combination of flash nucleosynthesis and convective mixing makes modeling difficult (Brown et al. 2001). Regardless, in the analysis below, we first attempt to identify as many of the stars associated with this group as possible, and then examine the evolution paths they take. Because the evolution is seen most clearly in some of the ultraviolet datasets, we 

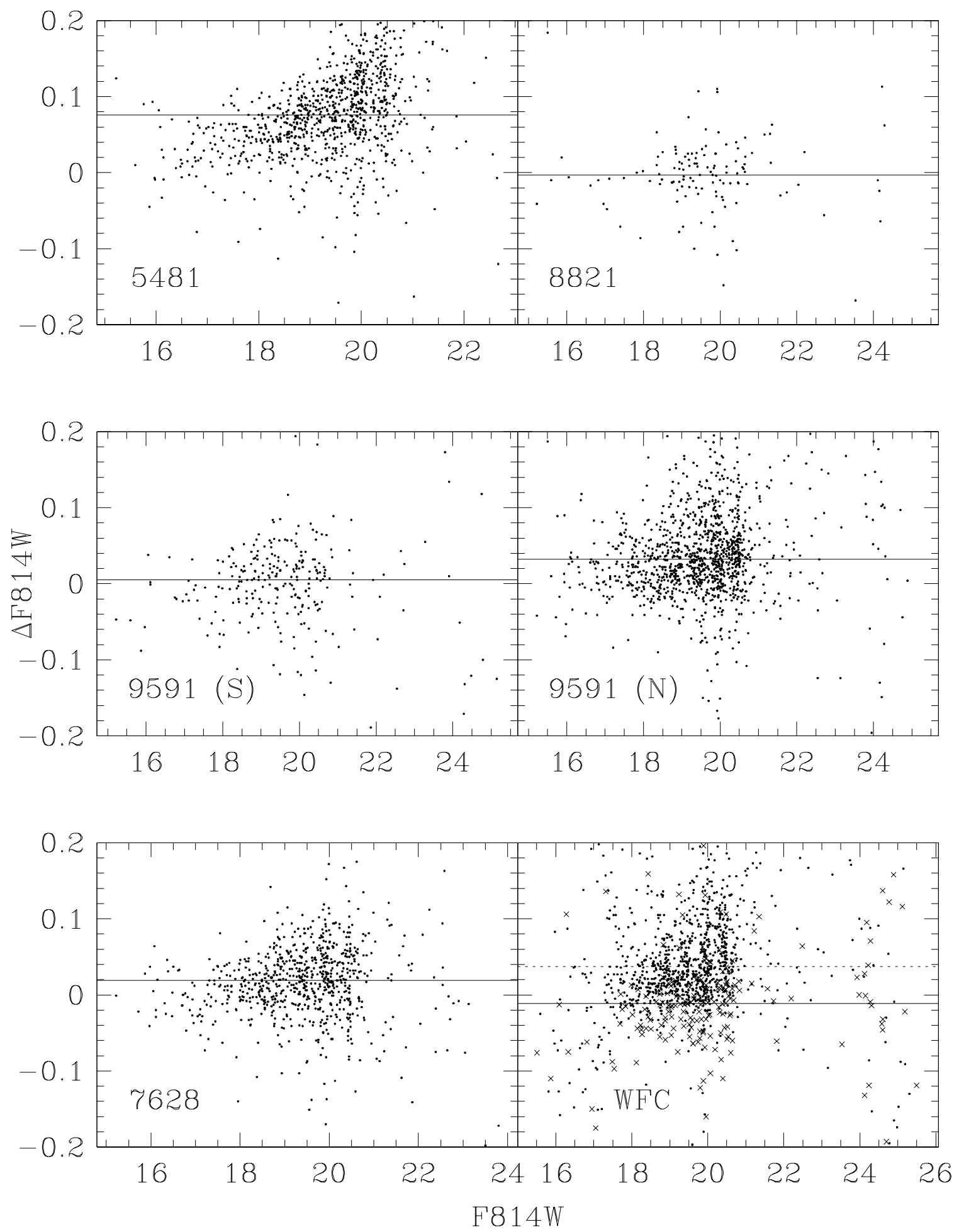

FIG. 7. - Residuals between F814W instrumental magnitudes in the sense of (7630 field - other). Lines show the median residuals. For the ACS WFC photometry, the solid line and $\times$ symbols are from the WFC2 chip (image extension 1 ), and dotted line and $\bullet$ symbols relate to the WFC1 chip (image extension 2). 


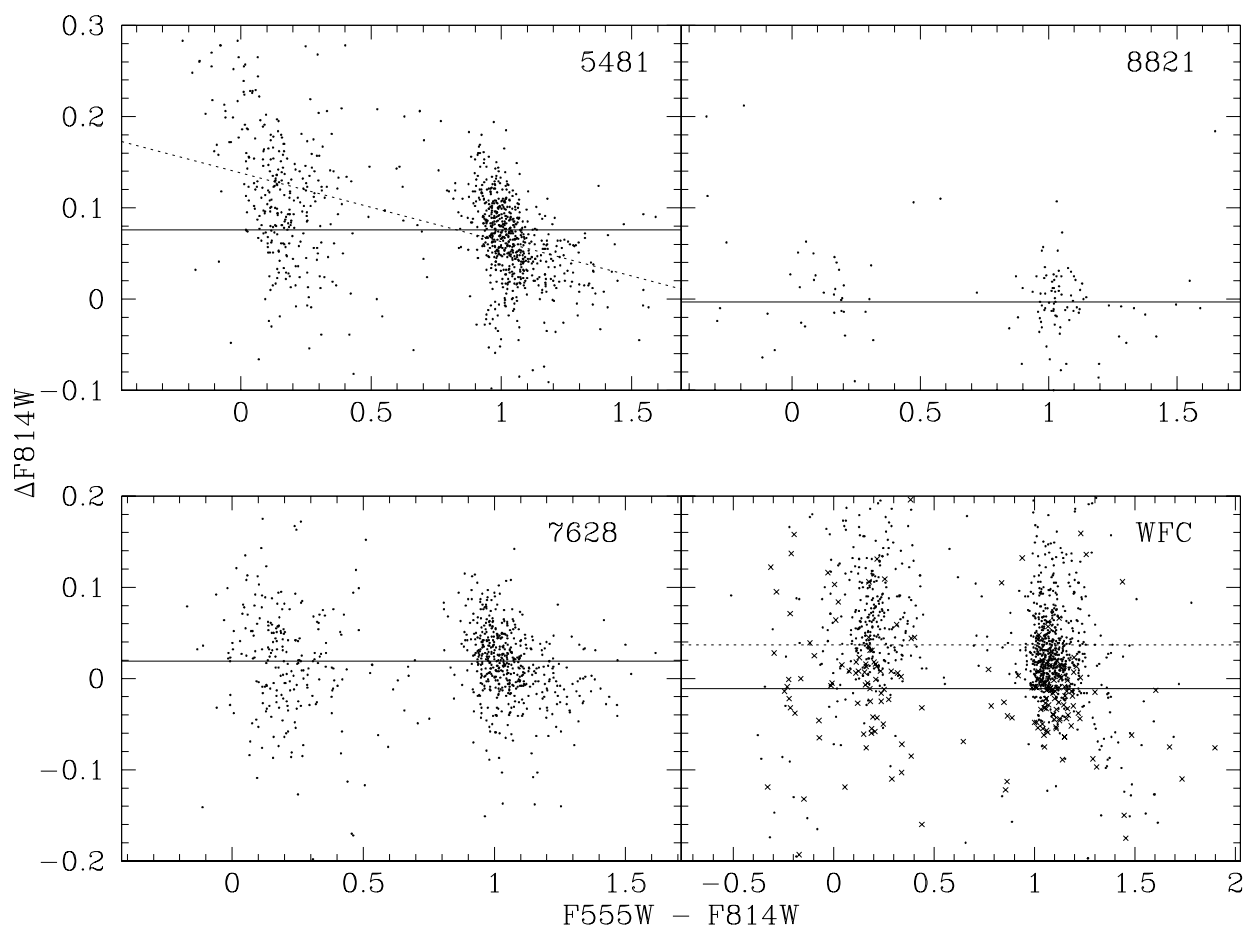

Fig. 8.- Residuals between F814W instrumental magnitudes in the sense of (7630 field - other). Points and lines have the same meaning as in Fig. 7 except that the fitted color-dependent trend in the 5481 data is shown.

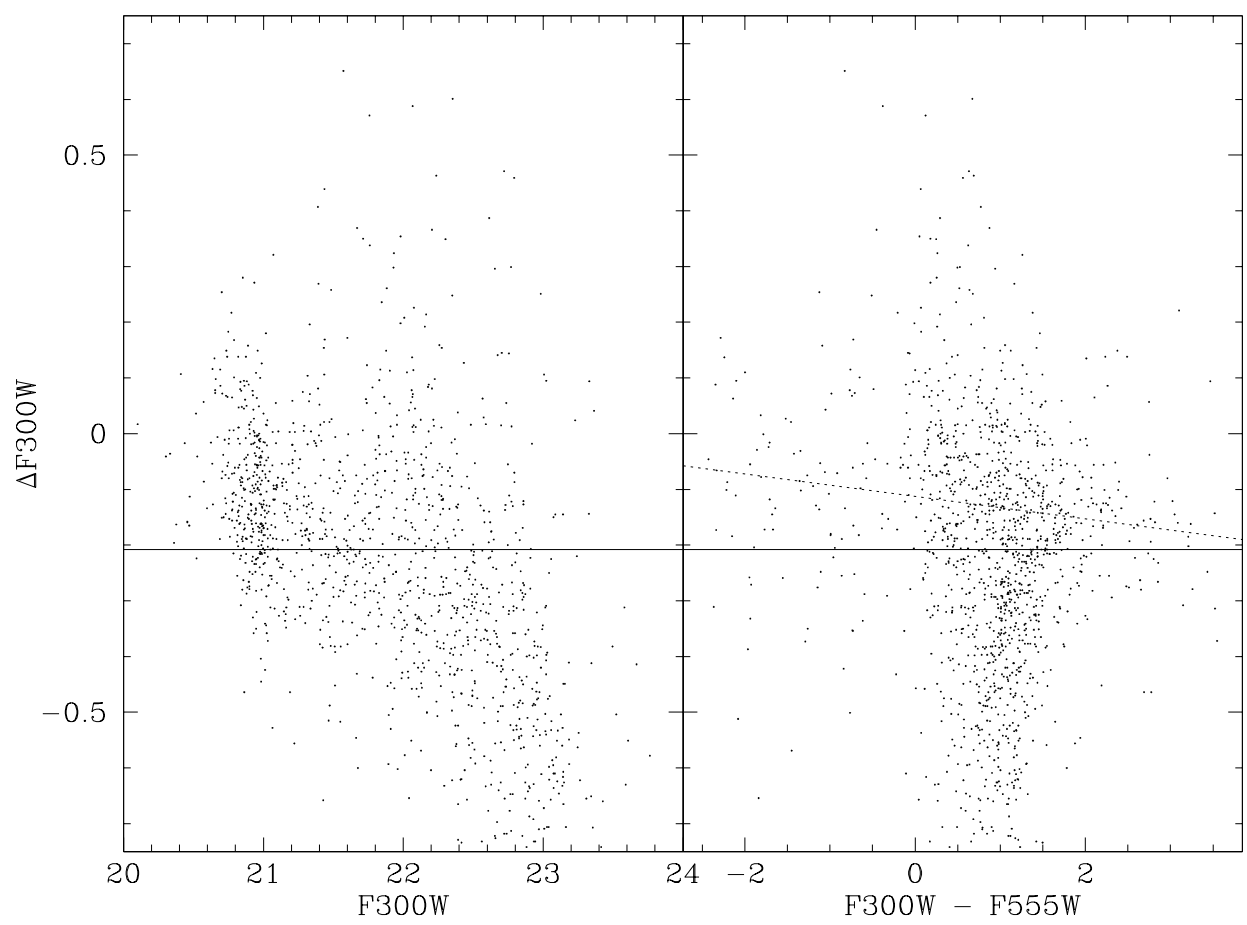

FIG. 9. - Residuals between F300W instrumental magnitudes in the sense of (7630 field - 7628 field). Solid lines show the median residuals, and the dotted line shows the fitted trend with color. 


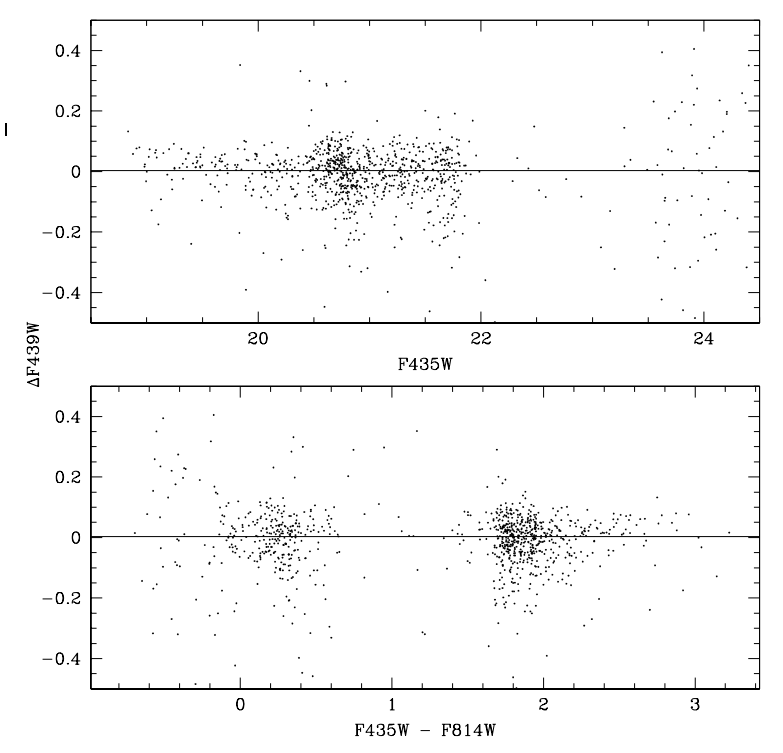

FIG. 10.- Residuals between WFPC2 F439W photometry (proposal 7628) and transformed ACS WFC F435W photometry in the sense of (WFPC2 - WFC). The solid line shows the median residual. Zero point differences between the WFC chips were less than $0.004 \mathrm{mag}$, and were not corrected.

discuss it in 93.2 .

In the ACS photometry (see Fig. 2), we consistently see a tail of stars stretching from the EHB/BHk group toward the faint end of the blue straggler group and main sequence turnoff $(23.6<F 435 W<25,-0.3<$ $F 435 W-F 814 W<0.5)$. These are probably stars that are optical blends of an EHB/BHk star with a main sequence star. Castellani et al. (2006) saw similar features in their optical CMD of NGC 2808. The features were not as strong there, probably because NGC 2808 has a much smaller EHB population producing such objects in the CMD. Fig. 14 shows loci of blends of three EHB/BHk stars (marked by the faintest $3 \times$ symbols) with main sequence and giant branch stars [represented by a Dotter et al. 2007 (hereafter, DSEP) isochrone having age of $13 \mathrm{Gyr}$ and $[\mathrm{Fe} / \mathrm{H}]=-2.1$ that was fitted to the stellar locus]. Blends with turnoff stars fall near the blue straggler region near the kinks in the curves. While such blends could masquerade as blue stragglers in optical CMDs, they can be clearly identified in ultraviolet CMDs due to the much higher surface temperatures of the HB star.

We use the results of the artificial star tests (2.1) to correct the distribution of detected HB stars for incompleteness. We took the total sample of identified HB stars and only kept stars that were detected in either the ACS WFC or HRC photometry and passed the same quality cuts as the artificial stars. This sample was made up of 856 stars, and with incompleteness corrections, the total sample expected in the field was 954. The top panel of Fig. 15] shows the detected and incompleteness-corrected HB distributions for the ACS fields. Based on this, the EHB population makes up more than $38 \%$ of the total HB population.

\subsubsection{Accounting for HB Star Lifetimes}

Theoretical models uniformly predict that stars at the blue end of the HB have 20-30\% longer HB lifetimes than stars near the instability strip, so we need to correct for this effect to determine how frequently EHB stars are being produced compared to the rest of the HB population. Because the $\mathrm{HB}$ population is used in determining the initial helium abundance, corrections for differences in $\mathrm{HB}$ star lifetimes can make the measurements more accurate. Below we describe a method for correcting for variations in $\mathrm{HB}$ lifetimes. As much as possible, we wish to produce a correction that i) is independent of chemical composition so that composition variations or imperfectly known compositions do not introduce systematic errors, and ii) is based on observational quantities.

For stars with the same initial compositions, we find that the lifetimes vary in similar ways as a function of stellar mass and effective temperature, although the absolute value of the lifetime does depend on composition. (In the following we have defined the end of the HB phase as the point when the central helium abundance $Y_{c}=0.05$; see the lefthand panels of Fig. 16. Because HB stars evolve somewhat in temperature during this phase, we have used the temperature of the star when it is halfway through its HB lifetime as a representative $T_{\text {eff }}$. We can imagine more complex algorithms for determining this temperature, but even the use of the $T_{\text {eff }}$ at zero-age produced minor effects on the results.) Based on this behavior, we defined a weighting factor for each star in the HB sample:

$$
w_{i}=\frac{t_{H B}\left(\log T_{e f f}=3.85\right)}{t_{H B}\left(\log T_{e f f}\right)}
$$

The largest portion of the variation in HB lifetimes occurs on the blue tail for $T_{\text {eff }} \gtrsim 10^{4} \mathrm{~K}$ in stars with lowmass hydrogen envelopes. By choosing $\log T_{\text {eff }}=3.85$ (near the blue edge of the instability strip) as the normalization point, we can correct the blue HB lifetimes to values representative of variable and red $\mathrm{HB}$ stars, which are common in globular clusters. The variation of the weighting factors with $T_{e f f}$ is shown in the righthand panels of Fig. 16. There is very little variation in the weighting factors with variations in $[\mathrm{Fe} / \mathrm{H}]$, so that residual uncertainties are at the level of a few percent. Further, the weightings only remove the effects of position on the $\mathrm{HB}$, and do not reference the absolute value of the HB lifetime, which does depend on composition and physics inputs to the stellar evolution codes (e.g., the ${ }^{12} \mathrm{C}(\alpha, \gamma){ }^{16} \mathrm{O}$ reaction rate; Cassisi et al. 2003).

The weighting factors also vary little with $Y$ for $\log T_{\text {eff }}<4.2$, although the agreement gets worse at higher $T_{\text {eff }}$. Higher helium abundance produces HB stars whose lifetimes increase more rapidly with decreasing $T_{\text {eff }}$ than stars with lower helium. So for clusters with the majority of their HB stars having $T_{\text {eff }} \lesssim 16000 \mathrm{~K}$, the weighting factors are almost entirely independent of composition, and corrected HB star totals can be used to gauge helium abundance directly using the $R$ ratio. For clusters like NGC 2419 that instead have a larger fraction of their stars at higher $T_{\text {eff }}$, this is not as straightforward, but the $R$ value can still be used as a test of whether clusters stars have enhanced helium. In fact, if weighting factors having a canonical value of $Y \approx 0.25$ are used, then helium-enriched stars on the blue tail will 


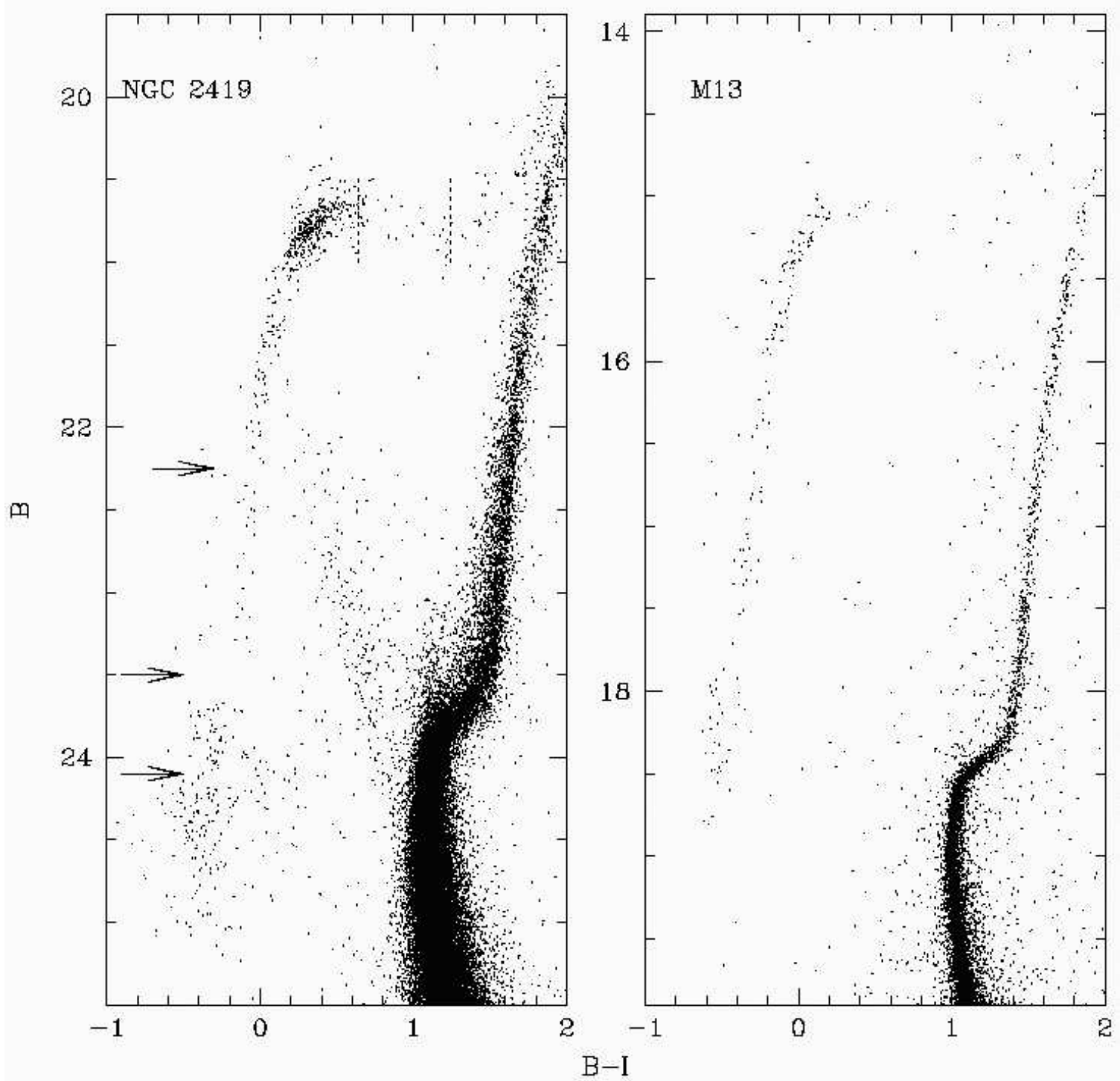

FIG. 11.- Comparison of the color-magnitude diagrams for NGC 2419 and M13 (Gordon et al., in preparation). NGC 2419 data comes from ACS WFC images, and F435W and F814W magnitudes were transformed to Johnson $B$ and Cousins $I$ using equations from Sirianni et al. (2005). Only the best measured stars $(|\mathrm{SHARP}|<0.5$, CHI $<5$, and crowding effects totalling less than 0.3 mag) have been plotted for NGC 2419. The data for M13 has been windowed to place the blue HB (near the instability strip) level with the corresponding portion of NGC 2419's HB. Arrows show the positions of possible gaps on the HB, and dotted lines show M5's instability strip (Sandquist \& Bolte 2004), shifted for the higher reddening of NGC $2419(\Delta(B-I)=0.18)$.

be undercorrected for their long lifetimes. This has the effect of leaving the corrected number of HB stars and the $R$ ratio too large, and implying a high $Y$.

The weighting has the most utility when dealing with stellar populations that have the same composition. Although NGC 2419 has not shown any evidence of large star-to-star composition $([\mathrm{Fe} / \mathrm{H}], Y)$ differences in its CMD (via large dispersion in its evolutionary sequences or multiple populations), other massive clusters show clear signs of internal chemical enrichment. Further, very blue HBs may result from helium enrichment. In order to look for signs of helium enrichment in NGC 2419 using the population ratio $R$, we need to correct for the effect of its blue $\mathrm{HB}$ first (in case it has a different cause, such as mass loss). Helium-enriched stars have longer lifetimes than unenriched stars at a given $T_{\text {eff }}$ at the blue end of the HB the difference is almost $10 \%$ for $\Delta Y=0.05$. Detection of a helium-enriched subpopula- tion of HB stars will still depend on the relative numbers of enriched stars, but the correction will provide a more accurate idea of the size of such a population. Some authors have previously attempted to correct for the lifetime effect. Cassisi et al. (2003) made corrections to $R$ for clusters with blue $\mathrm{HB}$ tails by using an $\mathrm{HB}$ evolutionary timescale appropriate to the mean HB mass. For clusters with bimodal HB distributions, this can lead to significant error because the lifetime is not a linear function of mass. Castellani et al. (2007) attempted to improve their analysis of $\omega$ Cen by applying corrections to the HB population using "typical" absolute lifetimes for stars in three well-populated parts of the HB. We believe our method is a significant improvement in accuracy.

Although color- $T_{\text {eff }}$ relationships remain imperfect, it is much more reliable to derive $T_{\text {eff }}$ from photometry than it is to derive stellar mass (which is the only other plausible choice for a physically-based coordinate 

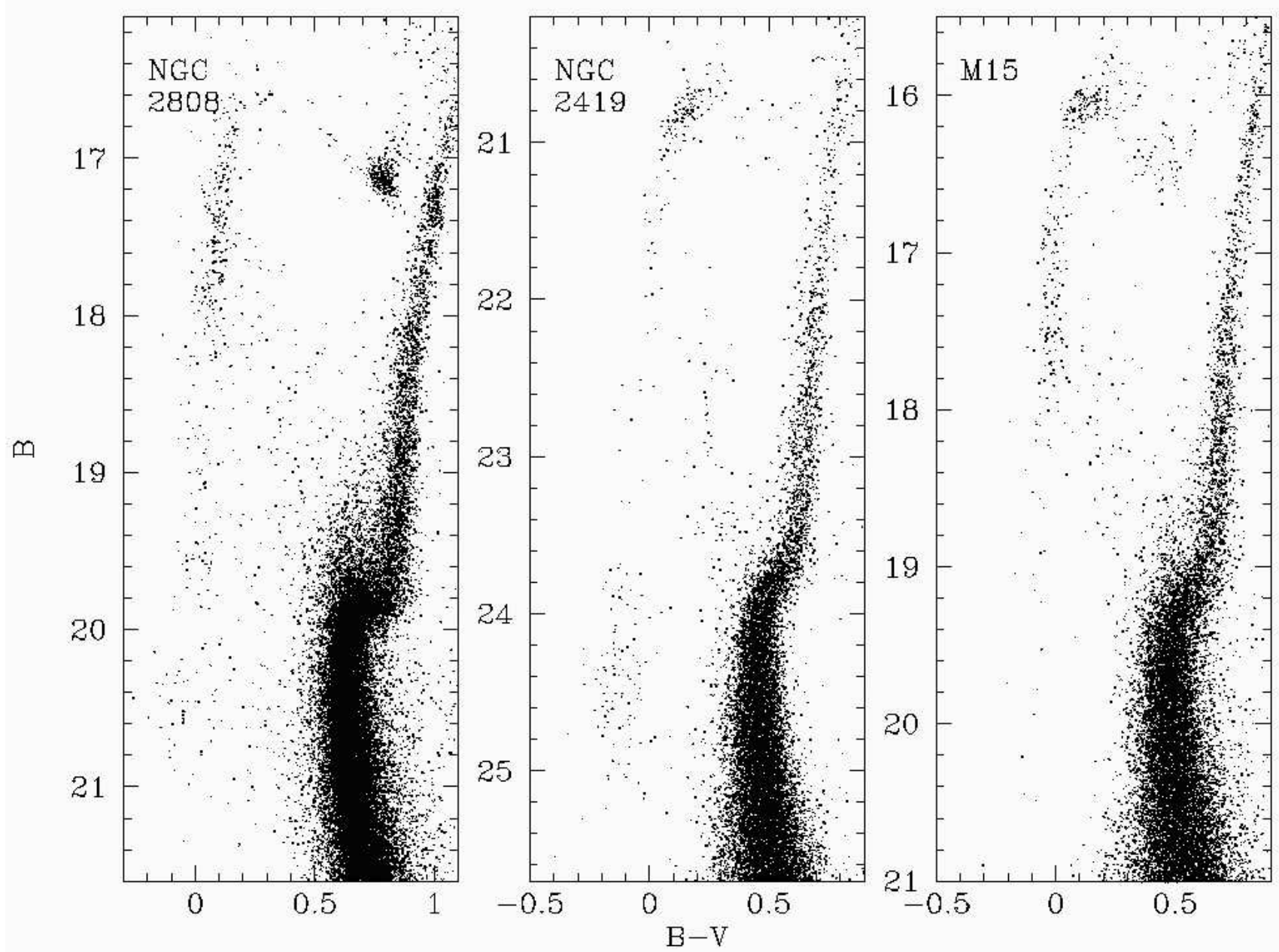

FIG. 12.- Comparison of the color-magnitude diagrams for NGC 2808, NGC 2419 and M15. NGC 2419 data comes from ACS WFC images, and was transformed to Johnson $B V$ using equations from Sirianni et al. (2005). The same quality cuts were applied to the NGC 2419 photometry as in Fig. 11 with an additional restriction on distance from the cluster center $\left(r<40^{\prime \prime}\right)$. Photometry for NGC 2808 (proposal ID 6095; P.I. Djorgovski) and M15 (proposal ID 5324, P.I. Yanny) comes from WFPC2 and was transformed using equations within HSTPhot (December 20, 2004 update; see http://purcell.as.arizona.edu/wfpc2_calib/2004_12_20.html). The data for NGC 2808 and M15 has been windowed to place the blue HB (near the instability strip) level with the corresponding portion of NGC 2419's HB.

describing position along the HB). To do this, we used DSEP HB models to determine an theoretical "average" HB line: specifically the locus of points halfway through the HB lives (ending at $Y_{c}=0.05$ ) of stars of different masses. The color- $T_{\text {eff }}$ transformation we used is shown in Fig. 17. This average line was fit to the stellar photometry and used to derive the offset between the theoretical and observed colors. For this cluster, we used the $F 435 W-F 814 W$ color (giving a color shift of 0.13 ) due to its good temperature sensitivity across the color range. Note that the evolutionary stage does not have a significant effect as long as the color and $T_{\text {eff }}$ are determined consistently - the other atmospheric variable affecting the colors (surface gravity) does not change greatly during the HB phase.

An additional benefit of using photometry to define the temperature scale is that there are features (such as the edges of the instability strip) that can be used as reference points. For example, the Grundahl $u$-jump has been found in many clusters with blue HB stars with $T_{\text {eff }} \gtrsim 11500 \mathrm{~K}$ (Grundahl et al. 1999). The $u$ jump is difficult to find in NGC 2419 because the HB is not heavily populated near its expected position, but in M15 it can seen at $F 336 W-F 555 W=-0.6$ (see Fig. 18). For NGC 2419, the jump appears to be at $F 336 \mathrm{~W}-F 555 \mathrm{~W}=-0.65$, which corresponds to $F 439 W \simeq 21.35$ in the WFC photometry, and a small color shift can be discerned at this position in the optical CMD. This is within the modestly populated blue wing of the main peak in the HB distribution. Our conversion from $F 435 W-F 814 W$ independently produced $T_{\text {eff }} \approx 10950 \mathrm{~K}$, gratifyingly close to $11500 \mathrm{~K}$ (Grundahl et al. 1999). ( $\left(T_{\text {eff }}=11500 \mathrm{~K}\right.$ was predicted to be about $0.05 \mathrm{mag}$ fainter.) In addition, the normal HB also seems to have a reasonably well-defined termination near $29000 \mathrm{~K}$ (Brown et al. 2008). As seen in Fig. 11, the end of M13's HB (which appears to correspond to the end of its hot $\mathrm{HB}$ ) translates to the middle of the EHB clump in NGC $2419(F 435 W \approx 24)$. Our color-temperature conversion again produces temperatures that approximately $(\sim 28500-29000 \mathrm{~K})$ match the theoretical value. A temperature of $35000 \mathrm{~K}$ (corresponding to the transition in atmospheric helium abundance spectroscopically observed in NGC 2808 and $\omega$ Cen) would fall near $F 814 W=24.9$ and $F 435 W=24.5$.

As expected from the analysis above, the use of dif- 


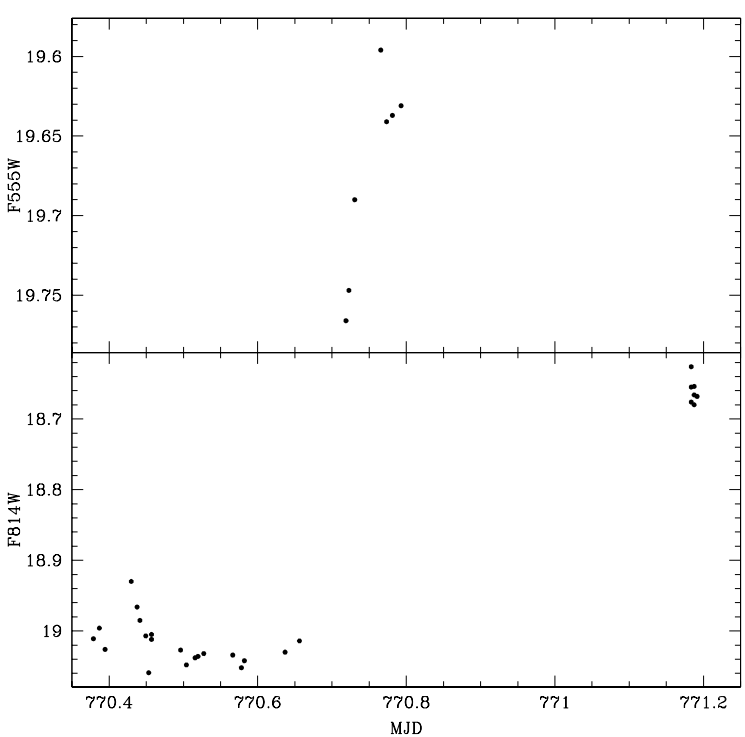

FIG. 13. - The light curve for the bright RR Lyrae variable V2 from $H S T$ photometry from proposal ID 7630. The time coordinate is modified Julian date (heliocentric Julian date minus 2450000).

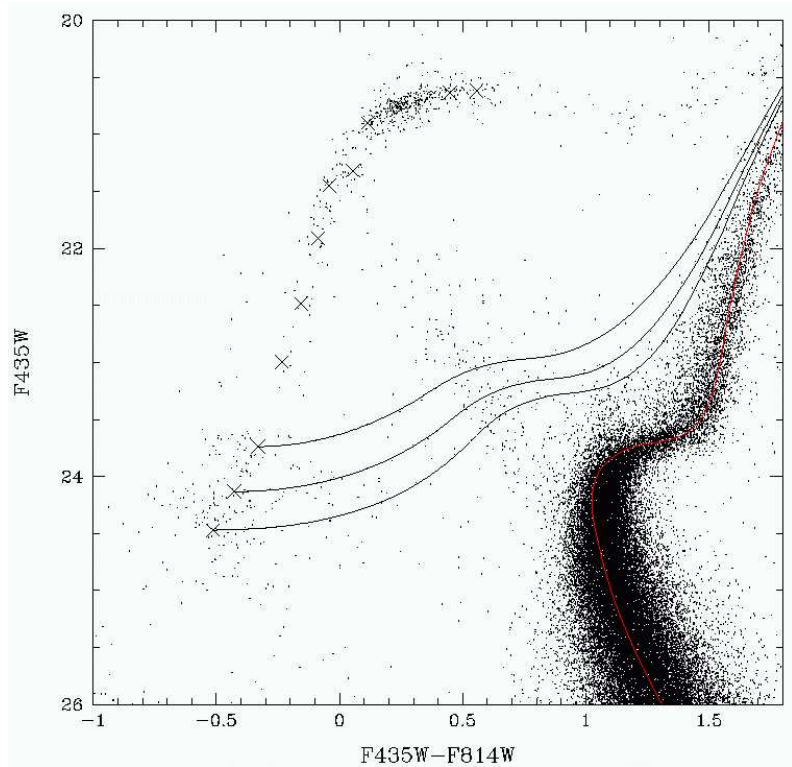

FIG. 14. - The effects of blending on the observed properties of EHB/BHk stars in the ACS WFC data. A DSEP isochrone (Dotter et al. 2007) with age $13 \mathrm{Gyr},[\mathrm{Fe} / \mathrm{H}]=-2.1$, and empirical color transformations was fit to the main sequence and lower giant branch (red line), and the curves show blends of the EHB star with a star whose properties were drawn from the fitted isochrone.

ferent sets of models will have a small effect on the lifetime-corrected HB star total. For our final analysis, we fitted a second-order polynomial to the weighting factors computed from DSEP models for $[\mathrm{Fe} / \mathrm{H}]=-2.0$, $[\alpha / \mathrm{Fe}]=+0.2$, and $Y=0.244$ in order to smooth out numerical noise. In addition, we assumed that stars redder than the main peak had evolved from the peak, and so were assigned weights from the main peak. We also needed to extrapolate the weighting corrections in the range $4.4 \leq \log T_{\text {eff }} \leq 4.5$, due to a lack of tabulated models. This is somewhat risky because a discontinuous

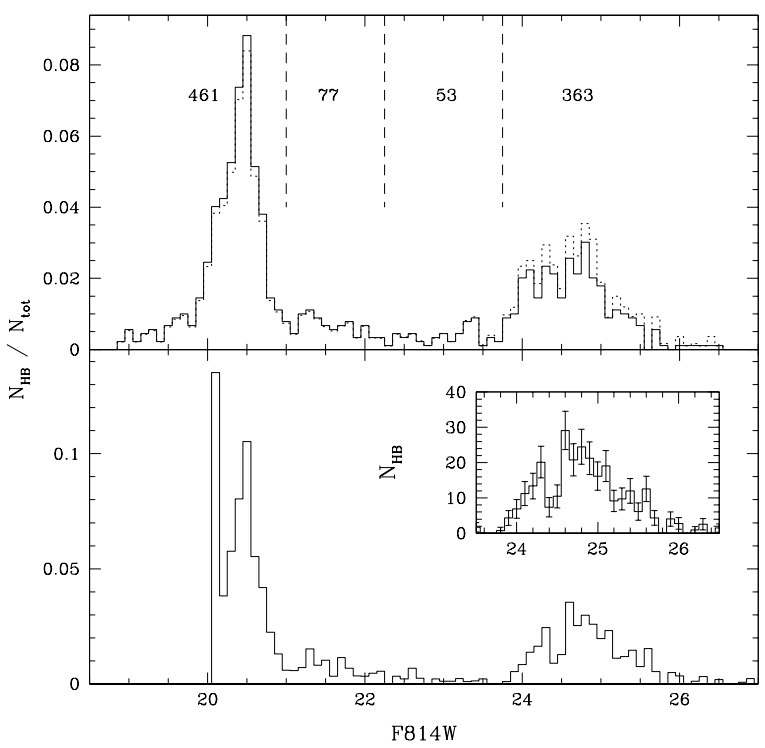

FIG. 15.- Top panel: Detected (solid line) and incompletenesscorrected (dotted line) HB star distributions for stars in the ACS fields. The numbers refer to incompleteness-corrected numbers of stars in each of the magnitude ranges separated by dashed lines. Bottom panel: HB star distribution corrected for lifetime differences, and including deprojections of evolved stars from the main peak $(F 814 W=20.1)$ and probable EHB/MS blends. Inset: Number of EHB stars, corrected for incompleteness and blending, and including Poisson uncertainties on each bin.
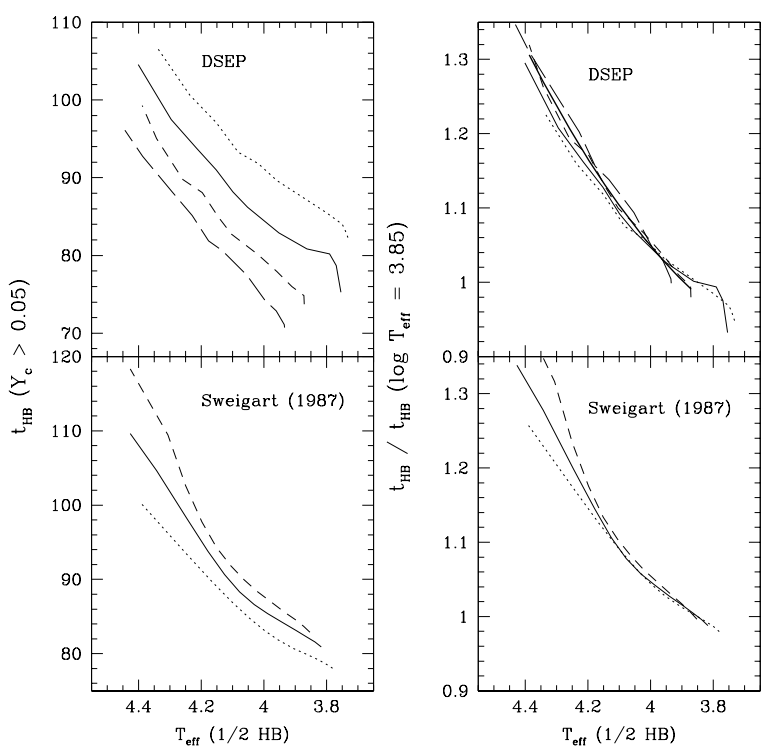

FIG. 16.- HB lifetimes as a function of $T_{e f f}$ from DSEP (Dotter et al. 2007) models for (from top to bottom) $[\mathrm{Fe} / \mathrm{H}]=$ $-1.0,-1.5,-2.0$, and $-2.5([\alpha / \mathrm{Fe}]=+0.2)$, and Sweigart (1987) models $Y=0.30,0.25$, and $0.20(Z=0.0001)$. Left panels: lifetimes in Myr. Right panels: lifetimes normalized to the stars reaching the zero-age HB at $\log T_{e f f}=3.85$. (For the DSEP model with $[\mathrm{Fe} / \mathrm{H}] \leq-2.0$, the most massive models do not reach that temperature, and were normalized at the lowest effective temperature present in the model.) In the top right panel, the bold line shows the polynomial fit to the $[\mathrm{Fe} / \mathrm{H}]=-2.0$ models that was used in making lifetime corrections. 


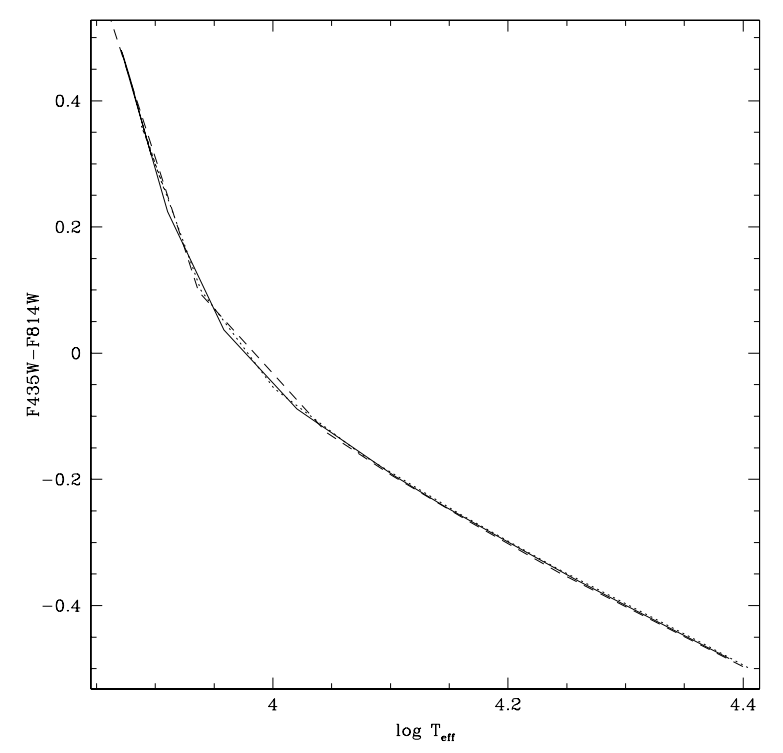

FIG. 17.- ACS WFC color- $T_{\text {eff }}$ conversion derived from DSEP (Dotter et al. 2007) models for $[\mathrm{Fe} / \mathrm{H}]=-2.0$ and $[\alpha / \mathrm{Fe}]=+0.2$. The lines correspond to the zero-age HB (dotted line), the HB termination (core helium $Y_{c}=0.05$; dashed line), and halfway in time between the two. No reddening has been applied to the color.

change in the chemical composition of the atmospheres (as there is in NGC 2808 and $\omega$ Cen) would cause a discontinuity in the temperature scale derived from the photometry. In this case we are likely to be underestimating $T_{\text {eff }}$ and the lifetime correction. This would produce a slight bias toward detecting a helium enhancement, but our corrections should still remove the lifetime effect to first order. With corrections for both incompleteness and differences in lifetimes, our original sample of $968 \mathrm{HB}$ stars becomes $N_{H B}^{\prime}=821 \pm 29$. (The quoted error bar is based on Poisson statistics for the corrected HB number.) It is important to note that this would correspond to the number of $\mathrm{HB}$ stars relative to bright RGB stars in our sample if i) the HB stars landed on the ZAHB near the blue edge of the instability strip, and ii) the initial chemical composition of all stars was the same. For this reason, it is appropriate to use this in the helium indicator $R$ (see 3.5 ).

If the stars were formed with the same composition, then we can use the lifetime-corrected histogram to derive the production rate of EHB stars relative to other HB stars: $33 \%$. However, increased metal and helium abundances both increase the HB lifetimes, so any chemical enrichment among some fraction of the cluster's stars would increase $N_{H B}^{\prime}$ above the value for a chemically uniform population. So the fractional production rate of EHB stars in NGC 2419 is at most 33\%. Fairly large changes in composition are theoretically predicted to produce fairly small changes in $\mathrm{HB}$ lifetime though: from DSEP models, a 0.5 dex change in $[\mathrm{Fe} / \mathrm{H}]$ changes the lifetime by about 5\%, and from Sweigart (1987) models a 0.05 change in $Y$ produces a $7 \%$ change among stars at the hot end of the HB. Only extreme chemical enrichment among the EHB stars is likely to mean that fewer than $25 \%$ of $\mathrm{HB}$ stars being produced are being made EHB stars.
We also note that if EHB stars are not produced at a constant rate through a cluster's lifetime that an EHB population would take some time to equilibrate because production rates (relative to other HB stars) would not initially equal "destruction" rates (stars evolving off the EHB). EHB populations could be expected to linger an extra 30 Myr or so after the production mechanism (whatever it might be) is shut off.

\subsubsection{An EHB Gap?}

Brown et al. (2001) presented evidence that the faint gap in NGC $2808(B \approx 20$; see Fig. 12$)$ corresponds to a theoretically-expected gap between EHB stars and blue hook stars which ignite helium while on the white dwarf cooling curve. Moehler et al. (2004) find that the gap in NGC 2808 corresponds to a spectroscopic transition from stars with helium-poor atmospheres (brighter than the gap) to ones with helium rich atmospheres. They find that the brighter stars have temperatures and gravities consistent with normal HB evolution, and that helium has probably diffused out of their atmospheres. The fainter, helium-rich stars have properties consistent with a mixed envelope associated with a late helium flash. In addition, Moehler et al. found that stars in $\omega$ Cen show a transition in atmospheric helium abundance at the same $T_{\text {eff }}(\sim 35000 \mathrm{~K})$, and Moehler et al. (1997) found a faint HB star in M15 $(B=19.75)$ with a helium rich atmosphere, and this star also falls below the level of the gap in NGC 2808. However, Brown et al. (2001) also noted that a gap similar to the one in NGC 2808 does not clearly show up in the photometry of some of these same clusters, so we have examined this question again here.

Blue hook candidates have been identifed in several clusters through a comparison of CMDs. Photometry in the F255W filter appears to give some of the clearest evidence of BHk stars. Clusters like M13 and M80 have HBs that terminate approximately 1.4 magnitudes below the knee (the blue HB stars with maximum brightness in F255W), and thought to have EHB stars but no BHk stars (Ferraro et al. 1998). Dalessandro et al. (2008) used $(F 255 W, F 255 W-U)$ CMDs to compare NGC 6388 and M80, and show that NGC 6388 had a group of stars that were fainter than the faintest $\mathrm{HB}$ stars in M80 and were separated from the rest of the HB stars by a gap. Photometry for NGC 2419 in the F250W filter from HRC camera (Fig. 3) places the bright end of the EHB about 0.9 mag fainter than the knee and the presumptive EHB gap about $1.1 \mathrm{mag}$ below the knee. Because the F250W and F255W bandpasses are not identical and the photometry for these hot stars are sensitive to it, we need a more exact comparison.

We used the " $B$ knee" as a reference point to shift the CMDs of the different clusters, as did Dalessandro et al. (2008) in their comparison of NGC 2419 with $\omega$ Cen. The knee is essentially based on surface temperature. NGC 2808 's HB is not well populated at the knee, which could produce some error, but its red HB allows us to check that the alignment with M15 is not far off. The most clearly visible gaps in NGC 2419's HB distribution do not match up with those in other clusters when the $\mathrm{HB}$ "knees" are aligned, as in Figs. 11] and 12, The possible exception is a marginally visible gap at $B \approx 24.1$ $(I \approx 24.5)$ in NGC 2419. Its presence is masked in the upper panel of Fig. 15] because of the presence of prob- 


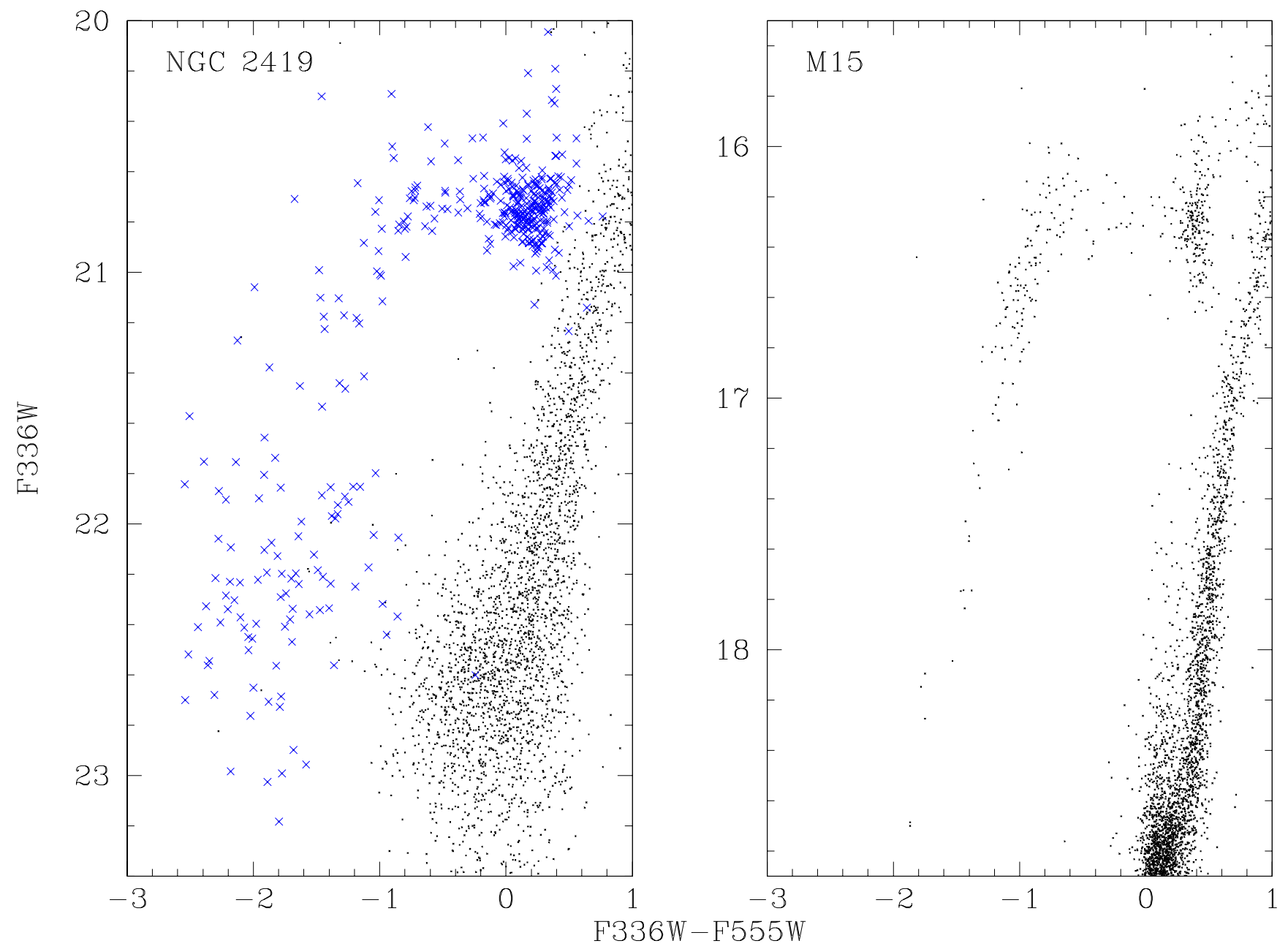

FIG. 18. - Comparison of the color-magnitude diagrams for NGC 2419 and M15 using the WFPC2 camera and F336W filter. The data for M15 has been windowed to place the blue HB (near the instability strip) level with the corresponding portion of NGC 2419's HB. Blue crosses are stars that were identified as HB stars via the methods described in 2

able EHB/MS blends. In the lower panel of that figure we have therefore deprojected stars back to the HB fiducial line using the blending lines shown in Fig. 14, After this procedure is executed as part of the incompleteness correction process, we see clearer evidence of a gap. The statistical significance of such a feature is hard to gauge because there is no theoretical expectation for how the stars should be distributed, but in the inset we plot corrected star numbers with Poisson distribution uncertainties. The bins at $F 814 W=24.4$ and 24.5 appear to be several standard deviations below the general trend defined by bins on either side. However, experiences with other clusters (Catelan et al. 1998) lead us to believe that there is a significant chance that it could be produced by statistical fluctuations even with the relatively large sample of stars we have in NGC 2419. However, NGC 2419 is not the only cluster to show a feature here.

As shown in Fig. 11. M13 has produced only a few possible HB stars hotter than the clump at the end of its blue tail (Ferraro et al. 1997), which terminates at the level of the weak gap in NGC 2419. In Fig. 12, M15 shows a scattering of stars in the EHB (the one spectro- scopically identified BHk candidate falls below the level of NGC 2808's gap). $\omega$ Cen has a large population of EHB stars with no gap, although Brown et al. (2001) mention that one might have been blurred by the wide metallicity dispersion from star to star. Still, a feature can be seen in the Castellani et al. (2007) HB distribution for $\omega$ Cen at $B \sim 18.5$. The distributions for $\omega$ Cen and NGC 2419 are compared side-by-side in Fig. 4 of Dalessandro et al. (2008), shifted in the same way as in Fig. 12. The feature appears there not so much as a gap, but as a strong contrast in CMD density: the fainter blue hook stars are much more common than the slightly brighter EHB stars. Using $V$-band and shifting the CMDs to account for differences in distance and reddening, Rosenberg et al. (2004) found that a gap on the extreme blue tail of M54 precisely overlaps the one in NGC 2808. The end of the theoretical zero-age HB fitted to NGC 2419 in Fig. 20 also appears to terminate at the approximate position of the apparent gap. All of this leads us to postulate that the weak gap at $B \sim 24.1$ in NGC 2419 is real, and marks the boundary between the canonical HB and blue hook stars. If true, this fea- 
ture appears to be largely metallicity-independent, and its visibility subject to the vagaries of the processes distributing stars into the blue tail.

If the initial chemical compositions of the bluest $\mathrm{HB}$ stars match those in the main HB peak, then we find that $25 \%$ of stars reaching the $\mathrm{HB}$ are fainter than this gap $(F 814 W>24.4)$ and may be blue hook stars. If they are more helium-rich, then this fraction would be reduced somewhat because we would have undercorrected for star lifetimes to get the distribution in the lower panel of Fig. 15. The additional correction is very unlikely to change the fact that this fraction is considerably larger than in any other cluster with the possible exception of $\omega$ Cen. We will return to this subject in the conclusions.

\subsection{Ultraviolet Bright Stars}

The source of the UV upturn at wavelengths shorter than $2300 \AA$ in spectra of elliptical galaxies remains an unsolved problem in stellar populations. The leading candidates for this emission are stellar types that have an appropriate combination of high surface temperature and relatively long lifetime. This includes extreme HB stars and their evolved forms, the AGB manqué stars; post-early AGB stars, which evolve to the AGB, but leave again before having a thermal pulsation phase; and post-AGB stars, which are short-lived but have high luminosity. The details of the evolution depend largely on the mass of the envelope outside of the fusion shells. Recently Brown et al. (2008) produced a UV CMD of the nearby elliptical galaxy M32, and found that current evolutionary models predict many more UV-bright post-HB stars than are observed.

NGC 2419 provides an interesting case study because of its large mass (which helps to populate relatively short evolutionary stages) and because the HB distribution can be described to first order with two strong peaks, as discussed in 3.1 . Our simple expectation is that the peak just to the blue of the instability strip is responsible for the majority of the AGB stars observed in the cluster. However, an examination of the AGB population may reveal whether the AGB stars evolve to a thermal pulsation termination, or whether they leave "early" at a lower luminosity level on the AGB. Further, a census of stars brighter than the $\mathrm{HB}$ and hotter than the AGB can help observationally identify how the EHB stars in this cluster evolve.

We have therefore used a combination of optical and UV photometry to identify probable hot post-HB stars. These are among the brightest objects in the F300W filter, as can be seen in Fig. 19. We first identified the two stars cataloged by Zinn et al. (1972) in their search for stars that were bright in both the ultraviolet $(U$, which is similar in bandpass to the F300W filter) and visible $(V)$. ZNG 2 is more than 30 times brighter than the $\mathrm{HB}$ in the F300W filter, and ZNG 1 is approximately 1.2 mag fainter than ZNG 2 and is brighter in the optical. Both have optical photometry consistent with being in a hot post-AGB phase. We identified five other stars that sit closer to the AGB in the CMD. The star PA 4 has $F 300 W$ between ZNG 1 and 2 and is brighter in F555W than either ZNG star. Two stars PA 6 and PA 7 did not have any UV photometry, but occupy similar positions in the optical CMD. Star PA 5 is faintest in the UV because it is the coolest star. Star PA 3 is significantly fainter in optical bands, and may be an example of a post-early AGB star. Star PA 6 was saturated in the WFC images (the only field in which it was observed), but the saturated photometry is accurate enough to conclude that it is post AGB.

There are several reasonable evolutionary scenarios that could explain the CMD positions of at least some of these stars (see Fig. 4 of Brown et al. 2001 for examples of evolutionary tracks). As described earlier, an RGB star that loses most of its envelope can leave the RGB before having a core He flash, evolving rapidly toward higher temperatures at nearly constant luminosity. Such stars can have a late He flash that lands them on the blue end of the HB, or can become a He core WD. Alternately, stars that had normal HB and AGB phases can undergo $\mathrm{H}$ and $\mathrm{He}$ shell flashes that lead to large excursions in both luminosity and surface temperature. These stars would be identified as post-AGB (PAGB) or post-early AGB (PEAGB) stars. PAGB and PEAGB stars move mostly in $T_{\text {eff }}$ during later flashes, but can get several times less luminous than the TRGB for shorter periods of time. Post AGB evolution is more likely to explain most of these stars, but the possibility exists that one of the more luminous stars might be a post RGB star. We expect that spectroscopy would the easiest way of identifying such stars.

One of the clearer features associated with the evolution of the EHB stars is a group of stars with $-0.5 \lesssim$ $F 435 W-F 814 W \lesssim-0.3$ in the range $22<F 435 W<$ 23.4 (see Figs. 2 or 14 for example). We have verified that all of the stars to the blue of the inter-gap HB population $(-0.2 \lesssim F 435 W-F 814 W \lesssim 0)$ remain to the blue of the $\mathrm{HB}$ in all of the UV CMDs. In the shortest wavelength filter $(\mathrm{F} 250 \mathrm{~W})$, the stars remain a nearly vertical sequence, implying luminosity evolution from the EHB rather than slight temperature evolution from the inter-gap HB (see Fig. 3). The large size of the EHB population compared to the inter-gap population also argues in favor of evolution from the EHB post-HB evolution is quite rapid (on a Kelvin-Helmholtz timescale) in comparison to the HB phase itself, so that there are likely to be many fewer post-HB stars. The ratio of evolved $\mathrm{HB}$ candidates to inter-gap $\mathrm{HB}$ stars is only about 1-to-2, whereas it is about 1-to-35 when compared to the EHB population. Theoretical ratios of post-HB (helium shell fusion) to HB lifetimes are typically around 0.12 (Cassisi et al. 2003), and it is quite possible that not all of the EHB stars produce post $\mathrm{HB}$ stars in this part of the CMD. DSEP model tracks for $[\mathrm{Fe} / \mathrm{H}]=-2.0$ and $[\alpha / \mathrm{Fe}]=+0.2$ approximately parallel the line traced out by these stars in optical bands (see Fig. 20). Their $0.52 M_{\odot}$ track comes close to reproducing the star colors, although there are no corresponding HB stars on the slowest portions of the track (between the zero-age HB and the portion shooting toward higher luminosity after core helium exhaustion). The gap of about $0.5 \mathrm{mag}$ in the $F 435 \mathrm{~W}$ filter is expected to correspond to the rapid evolution between core helium exhaustion and the establishment of a stable He fusion shell. This may be useful as an envelope helium abundance indicator specifically for the EHB stars because the helium abundance affects the envelope mass for a given effective temperature, and the envelope mass affects the star's luminosity when the He shell is established (Brown et al. 

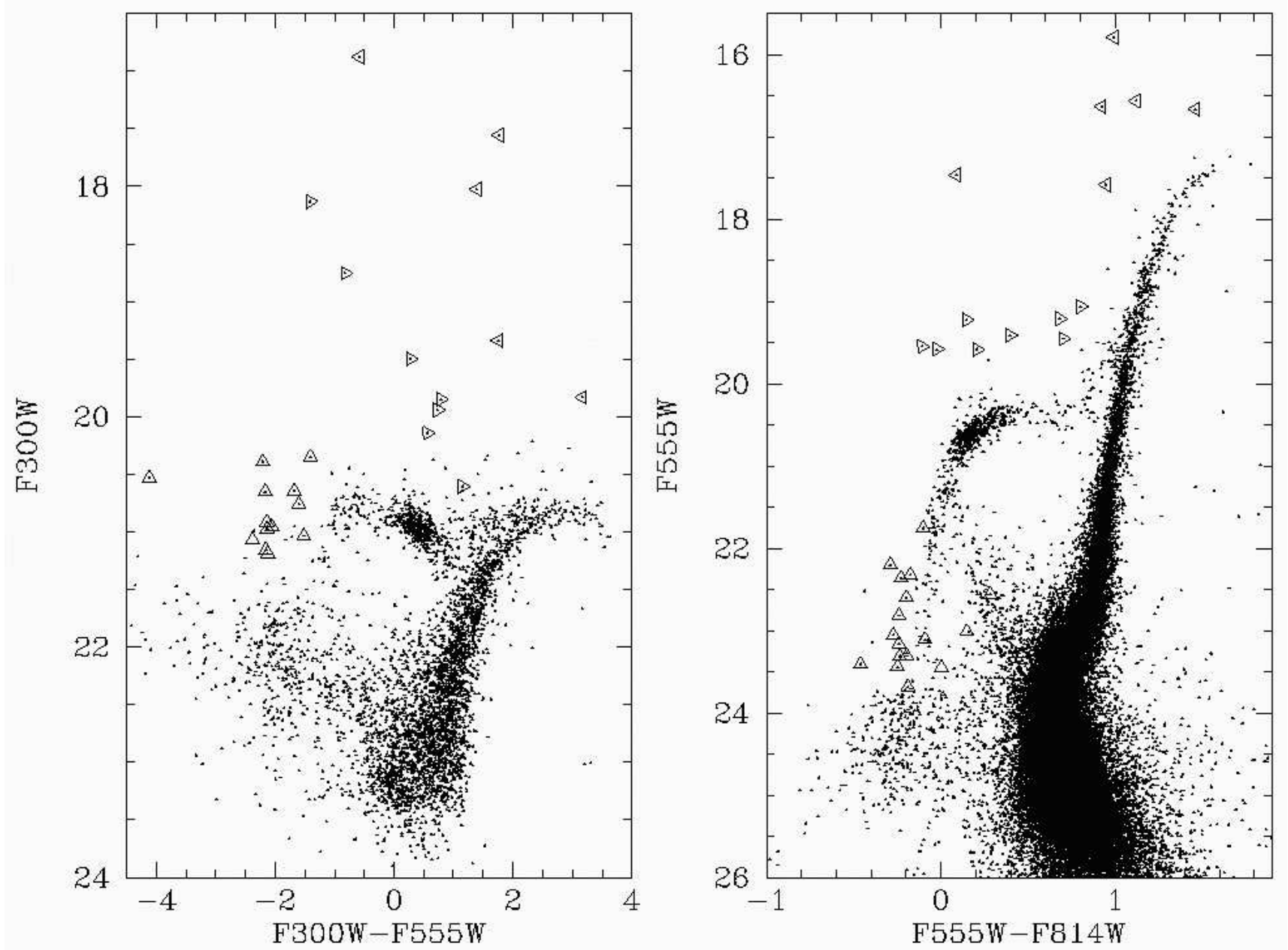

Fig. 19. - CMDs of ultraviolet-bright post-HB stars. The triangular symbols point in the expected direction of the evolution: upward for AGB manqué stars, rightward for supra-HB stars evolving toward the AGB, and leftward for post-AGB and post-EAGB stars.

2008). For more massive envelopes, the $\mathrm{H}$ fusion shell can be restarted at the same time, and this contributes significantly to the luminosity. At the moment, publiclyavailable models for EHB stars (evolved past central He exhaustion) with helium enrichment do not exist, so we are unable to calibrate this. We encourage such modeling because it may help answer questions related to the origins of multiple populations in clusters and the possibility that it is caused by cluster self-enrichment.

A second group of stars with $F 555 W \approx 19.5$ also appears to be composed of evolved HB stars. These appear to correspond to "supra-HB" stars identified in clusters like M13 and discussed in Strom et al. (1970) and Zinn (1974). The possibility that most of them are optical blends can be ruled out based on their brightness in the ultraviolet. (One star that falls in this range was identified as a blend in the HST photometry, however.) Among these stars is the bright RR Lyrae star V2, which was discussed in 3.1. We consulted theoretical HB models (Dotter et al. 2007; Cassisi et al. 2004) with very metalpoor compositions and no helium enrichment, and verified that stars originating from the primary peak in the $\mathrm{HB}$ distribution are expected to produce AGB stars exclusively - the evolution of the stars keeps them within about $0.1 \mathrm{mag}$ of the $\mathrm{HB}$ until central He exhaustion when they move to the AGB clump in about a Kelvin-
Helmholtz timescale. Models agree that as stellar mass decreases the post-HB evolution toward the AGB occurs brighter and brighter in $V$ until the tracks never reach all the way to the canonical AGB. In a small range of masses, stars can eventually evolve most of the way to the AGB, but spend a significant amount of time (more than a Kelvin-Helmholtz timescale) brighter than the $\mathrm{HB}$ at intermediate temperatures before reaching the AGB. Such a phase would correspond to the AGB clump if the stars had reached the canonical AGB. It is also related to the "bump" on the first ascent giant branch — during this time the He fusion shell comes into equilibrium after consuming the He abundance gradient left by the HB phase and adjusting to the new He fuel concentration.

A big question is where on the HB the supra-HB stars originate from. According to canonical models, the only evolution tracks that pass through this region come from inter-gap HB stars. However, the relative numbers of inter-gap stars and supra-HB stars appears to be inconsistent with this. Fig. 20 shows a comparison between our ACS WFC photometry and evolutionary tracks and synthetic HB populations from DSEP models. [We used DSEP products using their "empirical" color transformations as these appear to do a better job than the "synthetic" transformations in reproducing ACS data in and around the subgiant branch (Dotter et al. 2007; 


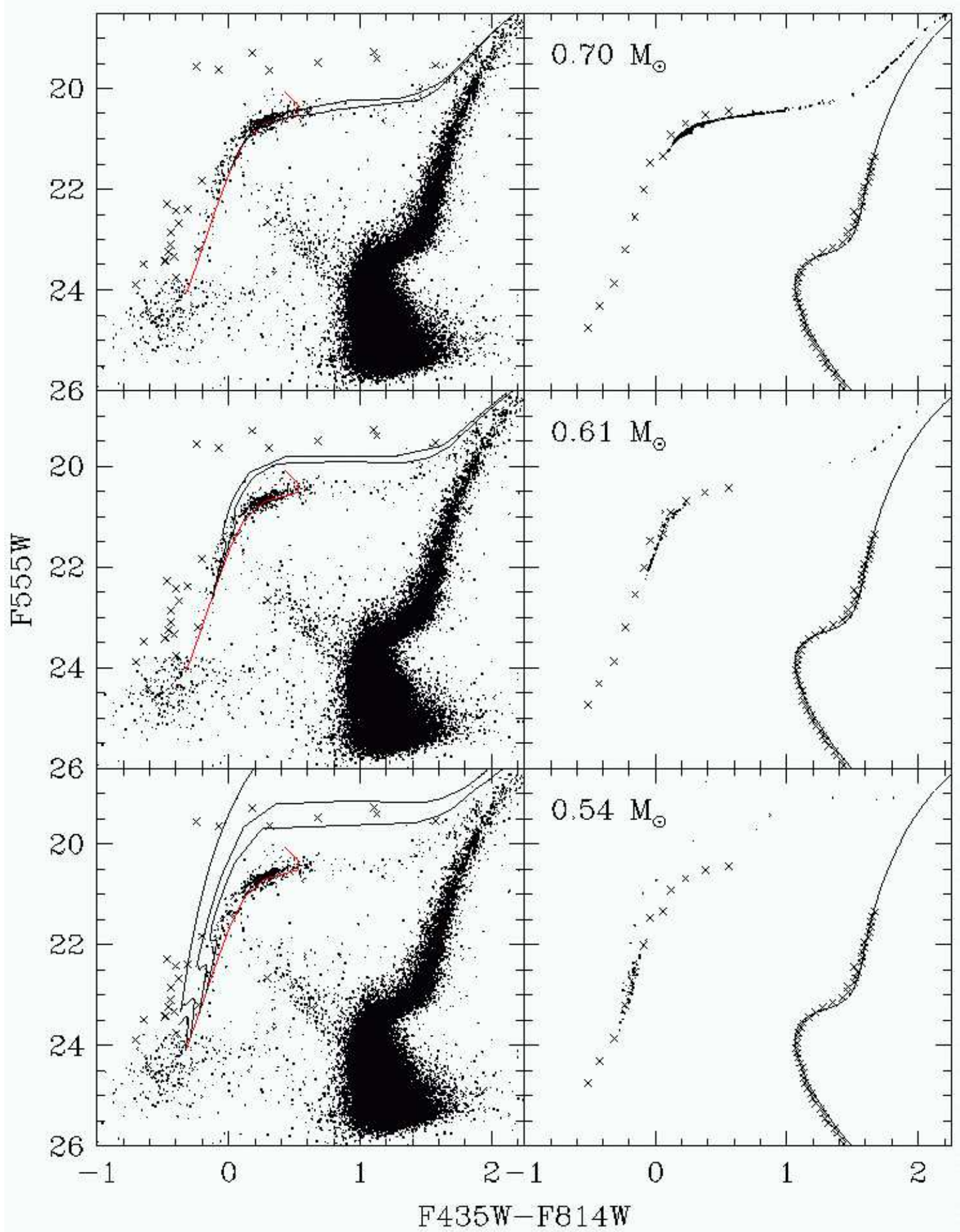

FIG. 20.- Comparison of ACS WFC photometry with DSEP HB models. In all panels theretical values are shifted by 19.97 in F555W and 0.2 in F439W-F814 to account for distance and reddening. Left panels: Comparison with evolution tracks having $[\mathrm{Fe} / \mathrm{H}]=-2.0$ and $[\alpha / \mathrm{Fe}]$ $=+0.2$. The ZAHB is shown in red. The tracks are for $0.51,0.52,0.54$, and $0.56 M_{\odot}$ (bottom panel), 0.58 and $0.6 M_{\odot}($ middle panel), and 0.65 and $0.7 M_{\odot}($ top panel). Right panels: Examples of synthetic horizontal branch populations having $[\mathrm{Fe} / \mathrm{H}]=-2.1,[\alpha / \mathrm{Fe}]=+0.2$, and a mass dispersion of $0.01 M_{\odot}$, The number of synthetic HB stars roughly corresponds to the number in the incompleteness-corrected sample (see Fig. 15). Also included (solid line) is an isochrone for the same composition and an age of 13 Gyr. Crosses show our mode fits to MS data, and individual stars that represent the mean HB line. 
Sarajedini et al. 2007).] The numbers of stars in the synthetic model representing the inter-gap population does not generate enough supra-HB stars (especially on the hot side), although it may explain the reddest stars in that group. Our best explanation for the number of evolved stars that are seen is that both the supra-HB and AGB manqué stars have evolved from the EHB peak, with the supra-HB evolving from the cooler (brighter in optical filters) part of the peak.

Increased envelope helium abundance tends to give stars a more pronounced blue loop during their early HB evolution, and they tend to retain higher surface temperatures during their subsequent evolution. (For examples, see Sweigart 1987.) Helium enrichment can also lead to a convergence of post-HB evolutionary tracks (see Fig. 9 of Busso et al. 2007 for an example) that could be responsible for supra-HB stars. For NGC 2419, an increase in envelope helium among the EHB stars might bring the models and observations into better agreement, but the increase may not need to be large since the theoretical tracks come close to matching the properties of the evolved HB stars. Once again though, theoretical models for EHB stars with varying helium abundance are need to settle the question.

\subsection{The Upper Red Giant Branch}

Because NGC 2419 has a significant population of EHB stars, it is natural to ask why these stars have been produced in such large numbers. In recent years, there has been growing evidence that subpopulations of chemically-enriched stars have been responsible for multimodal distributions of HB stars and for the existence of stars at the high temperature end of the HB. However, there has been no evidence of chemical composition variations within NGC 2419 from the CMDs (no large dispersion along the evolutionary sequences as seen in $\omega$ Cen, and no sign of multiple sequences), although it should be said, there has also been little in the way of spectroscopic abundance studies. As a result, we examined the possibility that extreme mass loss could be responsible for the bluest stars in the cluster. Helium enrichment within a stellar population does encourage the formation of blue HB stars by allowing lower mass stars to evolve off the main sequence and up the RGB. However, helium enrichment does not change the theoretical requirement that nearly all of the mass outside the helium core must be removed to put it on the EHB. If bright RGB stars deplete the mass stored in their envelopes before reaching the TRGB, they can avoid a traditional helium flash event, and instead may have a "hot flash" helium ignition that ultimately lands them on the EHB (see Fig. 4 of Brown et al. 2001, for example). Stars that can somehow lose their envelopes before reaching within about 0.4 magnitudes of the TRGB will not subsequently ignite helium, and are expected to produce He white dwarfs (D'Cruz et al. 1996; Brown et al. 2001).

Infrared excesses associated with giants in globular clusters provide evidence of dust formation, possibly associated with strong mass loss. Origlia et al. (2007) found excesses among a fraction of stars in the metalrich clusters 47 Tuc at luminosity levels down to the HB, and becoming stronger with luminosity. The presence of excesses among only a fraction of the stars implies episodic mass loss, although Bover et al. (2008) present

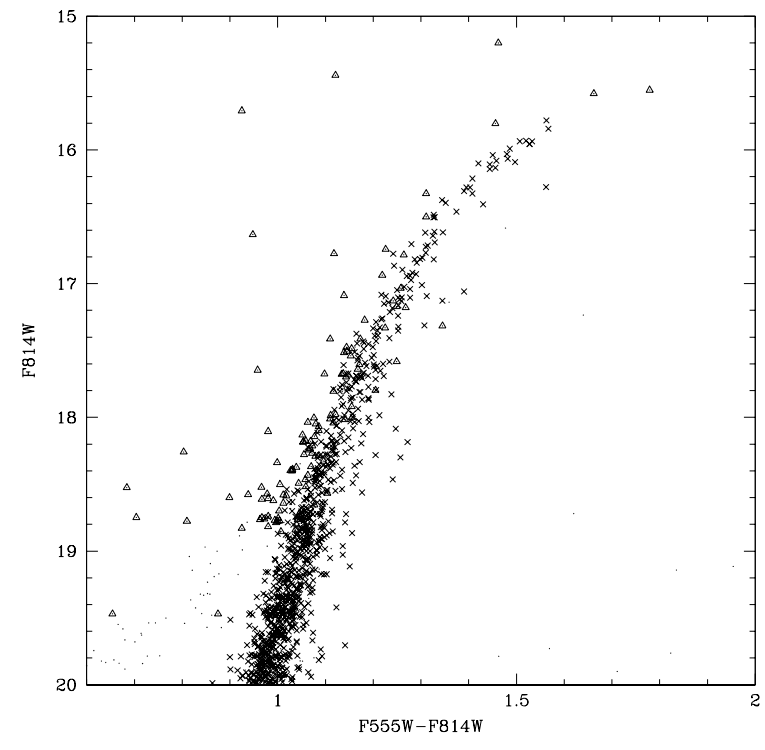

Fig. 21.- $(F 814 W, F 555 W-F 814 W)$ CMD for NGC 2419 stars on the bright RGB and AGB. The photometry is averages of measurements on the HST flight system after individual datasets were corrected for zeropoint differences relative to the proposal 7630 data.

evidence that much of this may have been due to blending in the infrared photometry. Boyer et al. find no evidence of mass loss below the TRGB in $\omega$ Cen, which is a cluster that is closer in composition to NGC 2419 that also has a large population of EHB stars. So at this point there is not strong evidence of strong mass loss below the TRGB.

We used the cumulative luminosity function in our analysis, counting stars starting at the observed TRGB. Comparison with models requires a magnitude shift and a vertical normalization. The horizontal magnitude shift was taken from the magnitude difference between the brightest observed red giant of the cluster and the TRGB in the theoretical model. By doing this, we virtually eliminate all dependences on age and chemical composition for $[\mathrm{Fe} / \mathrm{H}]<-1$ (Sandquist \& Martel 2007). It is partly for these reasons that the luminosity of the TRGB in $I$ has been used as a distance indicator for large, old metalpoor stellar populations. While there are giant stars that are brighter than the TRGB we identify, there is a large gap in $F 814 W$ that leads us to identify them as AGB stars, as shown in Fig. 21. The CMD also appears to be more uniformly populated fainter than the third brightest giant star having $F 814 W \sim 15.78$ that we identify with the TRGB.

For the vertical normalization we forced the models to have the same number of observed stars just brighter than the RGB bump at $F 814 W=18.4$. Shown in Fig. 22 are the observed data matched with models having $[\mathrm{Fe} / \mathrm{H}]$ of -1.84 and -2.01 . Although the $[\mathrm{Fe} / \mathrm{H}]=$ -1.84 model does the best job in matching the position of the RGB bump relative to the TRGB (see the inset in Fig. 221), in both cases the models predict a larger number of red giants than the observed data has between $F 814 W \sim 16.4$ and $F 814 W \sim 17.7$. As discussed by Sandquist \& Martel (2007), the shape of the cumulative luminosity function is a robust theoretical prediction.

The models were compared using the Kolmogorov- 


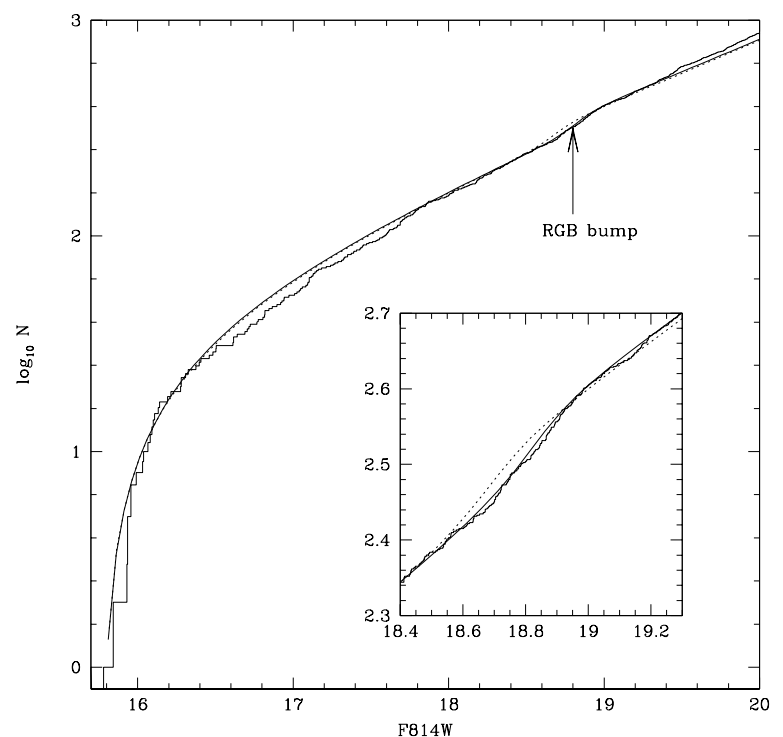

FIG. 22.- The cumulative luminosity function for bright RGB stars for NGC 2419, along with Victoria-Regina models (VandenBerg et al. 2006) for age $12 \mathrm{Gyr}$ and $[\mathrm{Fe} / \mathrm{H}]=-1.84$ (thin solid line) and -2.01 (thin dotted line) The inset shows a zoom centered on the position of the RGB bump.

TABLE 3

Cumulative Luminosity Function Comparisons FOR NGC 2419

\begin{tabular}{cccccc}
\hline \hline$\Delta F 814 W$ & $N$ & $D$ & $P$ & $D$ & $P$ \\
\hline & & \multicolumn{2}{l}{$[\mathrm{Fe} / \mathrm{H}]$} & $=-1.84$ & \multicolumn{2}{l}{$[\mathrm{Fe} / \mathrm{H}]$} & $=-2.01$ \\
1.0 & 41 & 0.102 & 0.769 & 0.097 & 0.817 \\
1.2 & 54 & 0.067 & 0.962 & 0.068 & 0.958 \\
1.4 & 72 & 0.101 & 0.434 & 0.100 & 0.441 \\
1.6 & 85 & 0.060 & 0.911 & 0.058 & 0.930 \\
1.8 & 102 & 0.048 & 0.970 & 0.046 & 0.981 \\
2.0 & 131 & 0.073 & 0.480 & 0.072 & 0.492 \\
2.2 & 154 & 0.055 & 0.721 & 0.054 & 0.744 \\
2.4 & 181 & 0.043 & 0.873 & 0.045 & 0.852
\end{tabular}

Note. - Col. 1: magnitudes below TRGB for cutoff of RGB sample. Col. 2: number of RGB stars in sample. Cols. 3, 5: absolute deviation between observed and theoretical cumulative luminosity functions. Cols. 4, 6: Probability from K-S test.

Smirnov (K-S) test. This evaluates the statistical probability that the null hypothesis (the two distributions are drawn from the same distribution function) can be disproven. The probability is based on the $D$ statistic, the maximum absolute difference between the cumulative distributions of the two sets. Although it has the advantage of making no assumptions about the precise form of the distributions, the probability that is calculated depends on the size of the sample. Because the choice of the cutoff magnitude for the comparison changes the sample size, we ran the K-S test with different cutoffs (essentially different normalization points). Table 3 shows the results of the K-S tests, including the number of stars brighter than each magnitude cutoff.

NGC 2419 only shows a slight deficiency of giants compared to the theoretical models. The minimum K-S test probability is $16 \%$ for a sample with $F 814 W \approx 17.9$. The maximum difference between the observations and models $\left(0.09\right.$ in $\left.\log _{10} N\right)$ seen at $F 814 W=16.6$ in Fig. 22 is comparable to the fraction of EHB stars relative to all HB stars: a 33\% decrease in the giant population would only correspond to a decrease in $\log _{10} N$ of about 0.12 . As a whole though, the results are marginally consistent with a population of giant stars having a distribution the same as the theoretical luminosity function. The results appear to validate the algorithms for computing plasma neutrino losses near the TRGB - Sandquist \& Martel (2007) noted that newer algorithms (e.g. Haft et al. 1994; Itoh et al. (1996) produce LF shapes that are consistent with observations. For typical RGB core conditions the rapid increase in neutrino energy loss is strongly dependent on the large temperatures and densities, and neutrino emission rapidly increases as the TRGB is approached. The shape of the LF would be changed if there were any energy loss mechanism having a significantly different dependence on temperature and density. The good agreement of the present NGC 2419 data with the models strengthens the case that the plasma neutrino losses are now being accurately modeled and nonstandard energy loss mechanisms are not operating in the core in a significant way.

The position of the modest deviation in NGC 2419's luminosity function also shows up at a different luminosity than the one noted in NGC 2808 by Sandquist \& Martel (2007). As noted earlier, stars within brightest 0.4 mag of the luminosity function are expected to ultimately produce HB stars: RGB stars having a normal helium flash at the TRGB will become normal HB stars, while RGB stars leaving the RGB a little early can have a late flash and become EHB stars. In NGC 2808, the brightest 1 mag in F814W seemed to be fairly uniformly below predictions by about 20\%. In NGC 2419 the brightest $0.6 \mathrm{mag}$ seems to be in good agreement with the models, while there are noticeable deviations in the next 1.4 mag. Even taking into account the longer lifetimes of blue HB stars having enhanced metal or helium abundances, the HB distribution implies that more than $25 \%$ of all HB stars being produced in NGC 2419 are becoming EHB stars (see 33.1 ). In NGC 2808, the fraction of HB stars that are on the EHB is significant, but quite a bit smaller (see Fig. 12, for example). Based on a simple interpretation of the RGB luminosity function, we would have expected NGC 2808 to have a large population of EHB stars, and NGC 2419 to have a relatively unpopulated EHB but a potentially substantial population of $\mathrm{He}$ white dwarfs. On the face of it, these results are somewhat contradictory. What could be happening in these clusters?

A first possibility is that there is unaccounted-for contamination of the RGB sample by AGB stars that masks a larger deficit of RGB stars in NGC 2419. EHB stars are not expected to evolve into stars that would be confused with RGB stars because they remain at high $T_{\text {eff }}$ in their post-HB evolution, but the main group of $\mathrm{HB}$ stars does produce AGB stars falling near the RGB. The bright RGB is particularly difficult to decontaminate because the AGB approaches the RGB most closely near the TRGB, and this is also the part of the RGB where contamination would have the largest effect on our ability to detect signs of RGB stars leaving early. More accurate photometry or spectroscopy would help to separate the 
AGB and RGB samples more definitively. However, our AGB star sample is quite large in comparison to the total HB population $\left(R_{2}^{\prime}=N_{A G B} / N_{H B}^{\prime}=0.146\right)$, and especially in comparison to the $\mathrm{HB}$ population in the main peak. So we believe we have identified the great majority of the AGB stars.

If the luminosity function shape is correct, is it possible that production of EHB stars does not require extreme mass loss? The primary suspect for accomplishing this is through a cluster's self-enrichment of helium. There is quite a bit of evidence supporting the picture of chemical self-enrichment within clusters, such as the sloping $\mathrm{HB}$ and unusually long periods of RR Lyraes in NGC 6441 (Caloi \& D'Antona 2007). There is also some evidence that chemical self-enrichment can reduce the need for dispersion in red giant mass loss to produce observed $\mathrm{HB}$ color distributions. For example, in M3 (which admittedly does not have EHB stars), Caloi \& D'Antona (2008) found that the combination of substantial color spread on the HB and a sharply peaked period distribution for RR Lyrae stars is most consistent with dispersion in helium abundance and virtually no dispersion in mass loss $\left(\leq 0.003 M_{\odot}\right)$. However, unless there is an extreme form of helium enrichment in the cluster, it would still be necessary for stars to lose nearly all of their envelopes to reach the EHB. An initial helium abundance $Y \sim 0.4$ would be required to reduce the mass of stars at the TRGB to $\sim 0.5 M_{\odot}$ (e.g. Newsham \& Terndrup 2007). An enrichment of this size should produce a clear second sequence in the CMD (remember that such a sequence would also need to have to be about $25 \%$ of the entire population). In NGC 2808, Piotto et al. (2007) identified a triple main sequence that was consistent with populations having different helium abundances ranging from primordial $(Y \approx 0.248)$ up to $0.35-0.4$. $\omega$ Cen shows a bluer main sequence (Bedin et al. 2004) that is more metal-rich than the majority of stars, and therefore is probably also helium enriched (Piotto et al. 2005).

If similar helium variations were responsible for the two HB populations in NGC 2419, a bimodal main sequence should be present in NGC 2419. In Fig. 23. we show a zoom on the main sequence in a high-quality subset of the ACS WFC photometry. A fiducial line was derived from stars in $0.1 \mathrm{mag}$ bins, and the main sequence was rectified by subtracting the color of the fiducial line interpolated to the magnitude of each star. Histograms of the MS star samples in $0.25 \mathrm{mag}$ bins are shown on the right side of the figure. The distributions resemble Gaussian distributions, and no feature appears consistently from magnitude bin to bin. A large chemically-distinct population can be ruled out with the present data unless there is a dysfunctional combination of helium and metal enrichment that cancels any color shift.

Other factors affect the interpretation of present-day samples of RGB and HB stars. For example, unless there is a physical process creating a semi-permanent feature in the luminosity function, an observed deficit of RGB stars (say associated with a statistical fluctuation) would propagate up the RGB, and eventually result in a reduction in the number of $\mathrm{HB}$ stars, although it should have no bearing on the issue of the production of EHB stars. The current sample of HB stars comes from stars that were on the RGB within about the last 130 Myr. According to models, a star moves through the uppermost $4.5 I$ mag of the giant branch (almost 4 mag in $V$ ) in $100 \mathrm{Myr}$, which is a typical lifetime for $\mathrm{HB}$ stars near the instability strip. For a population with a uniform composition and no mass loss, the stars in this range only vary in initial mass by about $0.002 M_{\odot}$ - a tiny range when translated back to the main sequence. In other words, the rapidity of RGB evolution should stretch out statistical fluctuations on the MS to the point of undetectability. The RGB cumulative luminosity function is also insensitive to changes in the total number of RGB stars in this range due to the way it is normalized. What Fig. 22 examines is variations in the numbers of stars at different luminosity levels within the sample. It is interesting that the two massive clusters with EHB stars that have been studied in this way have both shown small deficits toward the bright end of the RGB (and not excesses over theoretical predictions). However, the observed deficits appear at different luminosity levels (implying that different kinds of stars are being produced) and have marginal significance. In addition, only one cluster (M5; Sandquist \& Martel 2007) without EHB stars has been discussed in a similar manner, although it was consistent with theoretical expectations. We are forced to conclude that the different methods of examining RGB mass loss (detection of circumstellar dust and the luminosity function of the upper RGB) do not provide convincing evidence of strong mass loss well below the TRGB as yet.

\subsection{Radial Distributions}

Castellani et al. (2007) found trends in the radial distributions of stars in the massive cluster $\omega$ Cen, including a radial variation in the percentage of stars occupying the blue end of the HB. Structurally NGC 2419 and $\omega$ Cen are similar in concentration $(c=1.67$ for NGC 2419, and 1.34 for $\omega$ Cen; McLaughlin \& van der Marel 2005), although NGC 2419 has unusually large core and half-light radii $\left(r_{c}=8.7 \mathrm{pc}\right.$ and $\left.r_{h}=19.9 \mathrm{pc}\right)$ when compared to other clusters of similar luminosity $(4.1 \mathrm{pc}$ and $7.2 \mathrm{pc}$ for $\omega$ Cen, for example). However, when compared with other clusters of similar galactocentric distance, its core and half-light radii seem typical, but its total luminosity does not. We have a limited radial extent to examine here, but we have compared the cumulative radial distributions for the core sample in Fig. 24. A two-sample Kolmogorov-Smirnov test returns a probability of $55 \%$ that the RGB and bright $\mathrm{HB}$ stars are drawn from the same distribution, and of $47 \%$ for the RGB and AGB samples. So the RGB, AGB, and bright HB distributions are in reasonable agreement, with some small deviations of low significance.

\subsection{The $R$ Population Ratio}

Because the population ratio $R=N_{H B} / N_{R G B}$ is a sensitive indicator of helium abundance for clusters with large samples of evolved stars, we will attempt an additional test of the hypothesis that unusual CMD morphologies in the most massive clusters can be attributed to chemical self-enrichment.

The main difficulty in using the $R$ ratio is the calibration of the values. Recent models (Salaris et al. 2004) predict $R$ should have a value near 1.40 over a wide range of metallicities, but that the precise value depends on 

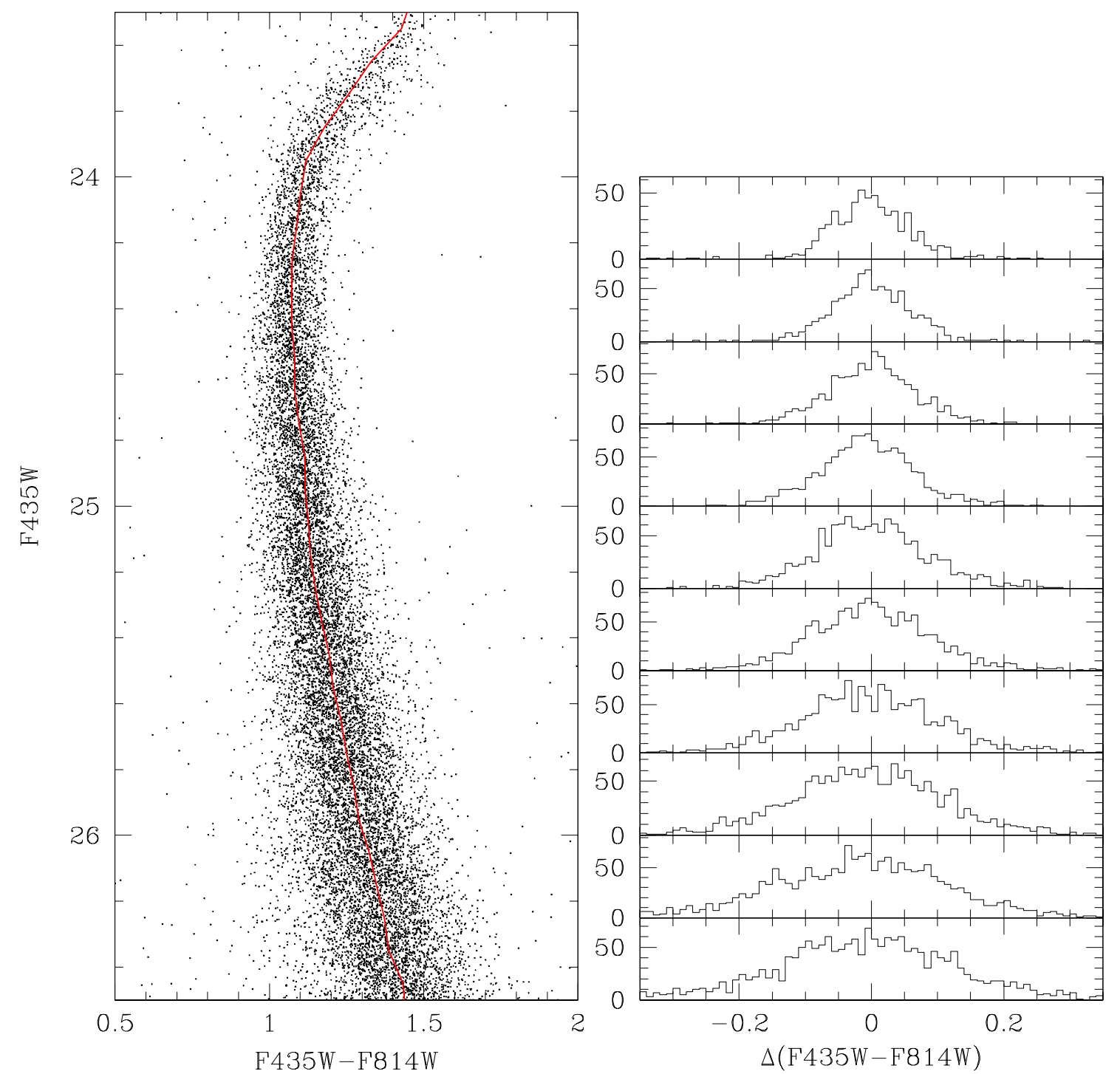

FIG. 23.- Left panel: The NGC 2419 main sequence in the WFC ACS dataset for stars with quality and radial position $\left(r<90^{\prime \prime}\right)$ cuts. Right panels: Histograms of stars from the left panel after the color of the fiducial line has been subtracted. The total sample is separated into 0.25 mag subsets.

the HB morphology. To make a reliable comparison of He abundances in a relative sense, we must correct for variations in lifetimes along the $\mathrm{HB}$. This correction is important for a cluster with a blue HB morphology like NGC 2419, and we discussed our method in 33.1 .

In order to compare NGC 2419 to other clusters, we follow a method similar to that described by Recio-Blanco et al. (2005) to select the faint limit for the RGB star sample. In their method, a template cluster having an intermediate HB morphology is fit to the cluster data and used to identify a reference HB level. Because NGC 2419 is a very metal-poor cluster, we used M68 (Piotto et al. 2002) as the template. The M68 photometry was shifted in magnitude and color to achieve the best possible fit. (As discussed in 33.1 and below, the red HB stars in NGC 2419 seem to exclusively be evolved HB stars, so we looked for the best fits with stars in the main HB peak and bluer.) The comparison between the proposal 7628 dataset (the only one that used the F439W and F555W filters) and the Piotto et al. (2002) dataset for M68 is shown in Fig. 25] with the M68 photometry was shifted by $4.75 \mathrm{mag}$ in $F 555 \mathrm{~W}$ and $0.05 \mathrm{mag}$ in $F 439 \mathrm{~W}-F 555 \mathrm{~W}$. The error in the magnitude comparison appears to be about the same as the 0.07 mag quoted by Recio-Blanco et al. (2005) in their work. Based on the reference ZAHB value for $\mathrm{M} 68\left(F 555 W^{Z A H B}=15.73\right.$; Recio-Blanco et al. 2005), this gives $F 555 W^{Z A H B}=20.48 \pm 0.08$ for NGC 2419 . Taking into account the zeropoint difference between the datasets for proposals 7628 and $7630(-0.017 \mathrm{mag}$ in F555W), we have $F 555 W^{Z A H B}=20.46 \pm 0.08$ on the reference system. The ZAHB level in F555W is used in the definition of the faint end of the RGB sample in the 


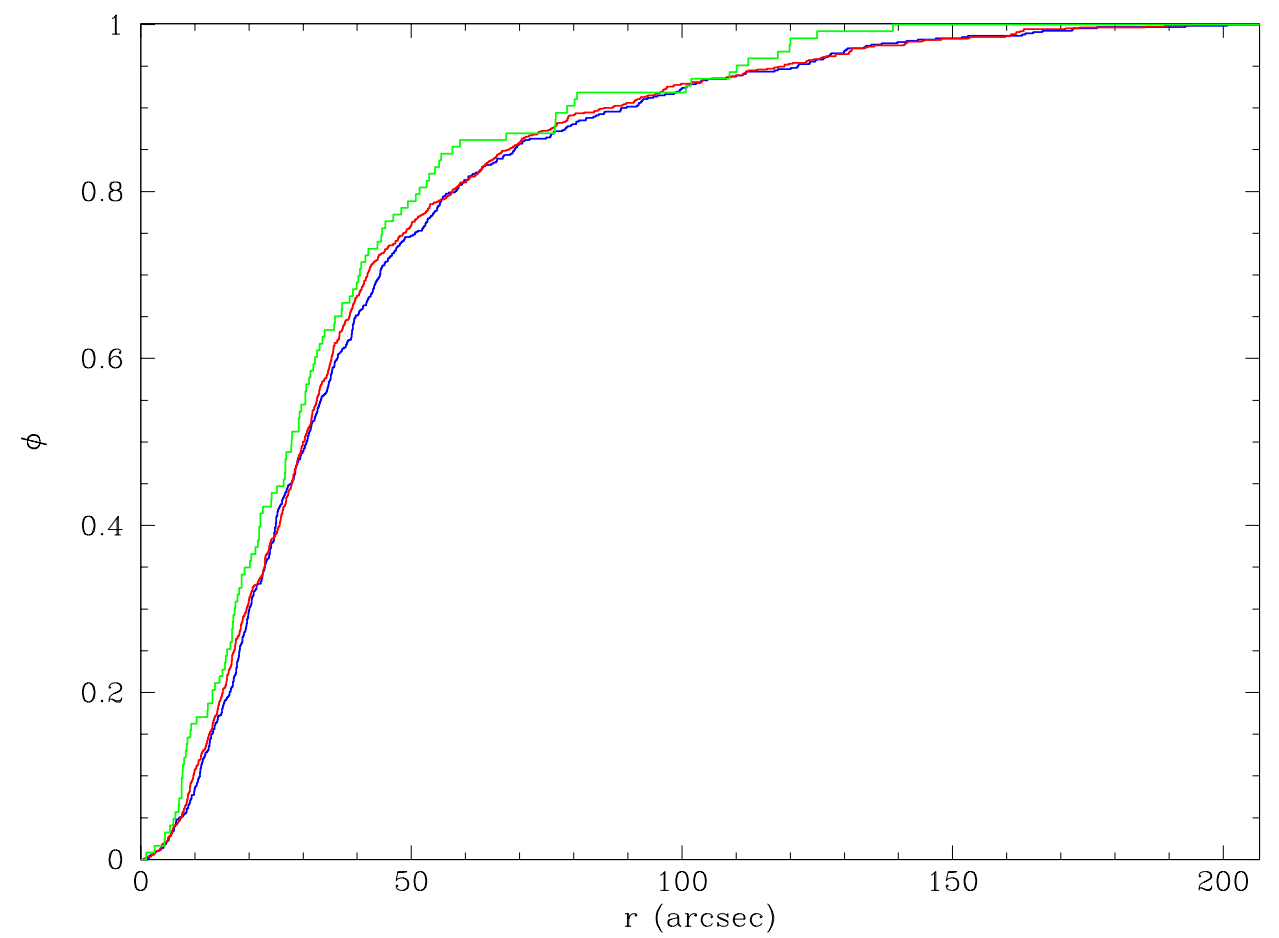

FIG. 24. - The cumulative radial distributions for evolved stars in the combined HST fields. The red line shows bright RGB stars $(F 814 W<20)$, green shows AGB stars, and blue shows HB stars excluding the EHB $(F 814 W<23.75)$

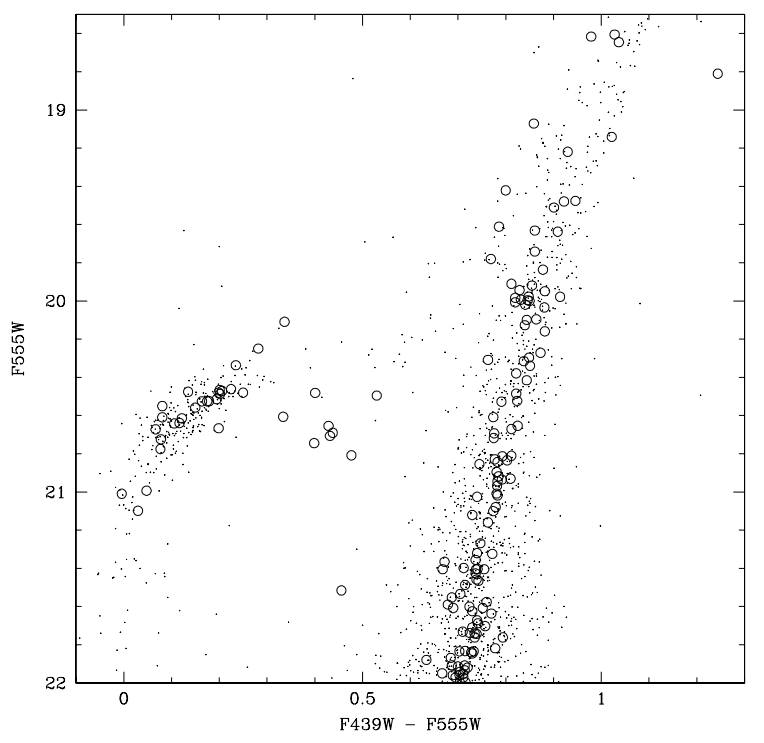

FIG. 25.- WFPC2 CMDs for NGC 2419 (dots) and M68 (open circles; Piotto et al. 2002). The M68 dataset has been shifted to match the blue HB of NGC 2419.

studies of Zoccali et al. (2000) and Salaris et al. (2004), and we have used the same definition here for the most direct comparison to other clusters.

With the zeropoint, we find $N_{R G B}=529_{-31}^{+47}$ for stars in the same fields as the HB stars (i.e. the ACS WFC fields of view). The quoted error bars on $N_{R G B}$ come from the uncertainty in the ZAHB level, which dominates Poisson statistics as a source of error. Although NGC
2419 has a fairly large number of RR Lyrae stars that could be used to establish the ZAHB level, indications are that all of the RR Lyrae stars have significantly evolved away from the blue HB (see \$3.1). Ripepi et al. (2007) find $\left\langle V_{R R}\right\rangle=20.31 \pm 0.01$ from 67 stars, but also find that the period-amplitude distribution is most consistent with the "well-evolved" RR Lyrae variables in M3. For the RR Lyrae stars in M68, Walker (1994) finds $\left\langle V_{R R}\right\rangle=15.64 \pm$ 0.01. The M68 RR Lyrae stars have positions in the period-amplitude diagram that are consistent with the "regular", relatively unevolved stars in M3. The implied magnitude shift between NGC 2419 and M68 from $\left\langle V_{R R}\right\rangle$ measurements is 4.67 mag. This is smaller than the value derived from CMD comparison, and does not produce an adequate fit. This again is consistent with the idea that the NGC 2419 RR Lyrae stars are evolved and do not provide a good estimate of the ZAHB position, so we therefore rely on the CMD fit value.

When combined with $N_{H B}^{\prime}=821 \pm 29$ (the HB sample when corrected for incompleteness and for lifetimes of blue HB stars relative to stars at the edge of the instability strip), we find $R=1.55_{-0.13}^{+0.09}$. The error quoted for $N_{H B}^{\prime}$ only includes Poisson errors, but the uncertainty in $N_{R G B}$ deriving from the ZAHB magnitude level dominates the uncertainty in $R$. For $[\mathrm{Fe} / \mathrm{H}]=-2.1$ and $Y=0.245$, Salaris et al. (2004) find $R=1.40$ from theoretical models, and this value changes by at most 0.01 at $[\mathrm{Fe} / \mathrm{H}]=-1.8$. This implies that the average $Y$ for NGC 2419 stars is higher than primordial by about 0.016 , but the errors indicate this is just above a $1 \sigma$ difference. This is in spite of at least two effects that probably produce systematic biases toward larger helium abundances (see \$3.1). (Without the correction for star lifetimes, the HB 
population would have been $N_{H B}=954$, giving $R=1.81$ and implying an average helium abundance enhancement of about 0.041.) Still, if the EHB population (about $31 \%$ of the corrected total) is the only part that is helium enriched, it could be enhanced by about 0.05. Based on the evolved stars, there is only weak evidence of helium enrichment.

As a final comparison, we mention the population ratios for $\omega$ Cen computed by Castellani et al. (2007). Though they did not compute an $R$ value that could be directly compared with ours, their comparisons of $\mathrm{HB}$ to RGB or MS stars implied that the HB star sample was 30-40\% larger than predicted by canonical evolution models. Their result was actually larger than would be expected given that $\omega$ Cen appears to host heliumenriched populations. Regardless of the reason for the discrepancy, it further underlines the weak evidence of helium enrichment in NGC 2419.

\section{CONCLUSIONS}

We have examined archival HST imagery in the optical and ultraviolet to study evolved stars in the core of the massive globular cluster NGC 2419. An important aspect of these populations is the clear separation of the horizontal branch population into two groups: the primary peak just to the blue of the instability strip and a secondary peak of comparable size at the extreme end of the HB. More than $38 \%$ of the HB stars in this cluster are in the EHB peak, and if their initial compositions were the same, this means that more than $33 \%$ of its red giants have been diverted into this population (more than $25 \%$ into blue hook candidates). While our study only examined the core population (and in $\omega$ Cen, for example, there is some evidence for a decrease in the abundance of EHB stars with distance from the cluster center), this is a feat that is even remotely matched by only some of the very most massive clusters in the Galaxy. For example, $\omega$ Cen has approximately $27 \%$ of its HB stars on the EHB (Dalessandro et al. 2008) - a slightly smaller proportion than NGC 2419. M54 also has a modest population of EHB stars Rosenberg et al. 2004; Siegel et al. 2007), but it is a significantly smaller fraction of the total HB population (we estimate $\lesssim 15 \%$ from the CMD in Rosenberg et al. 2004). NGC 2808 also has a modest EHB population that is $12 \%$ of its total $\mathrm{HB}$ sample (Castellani et al. 2006, where we have included their EBT3 stars and the HBp stars that are likely to be composed of blends of EBT3 and MS stars). In the very metal-rich cluster NGC 6388 (Dalessandro et al. 2008), only about $3.5 \%$ of the HB stars are on the EHB $(2 \%$ as blue hook stars). A few other massive clusters (e.g. NGC 6441, M15) have a handful of EHB stars that compose a few percent of their populations at most, and another massive cluster (47 Tuc) has no known EHB stars. $\omega$ Cen, M54, NGC 6388, NGC 6441, and 47 Tuc are probably the only clusters that are more massive than NGC 2419 (McLaughlin \& van der Marel 2005), while NGC 2808 appears to have a similar mass and M15 is approximately $50 \%$ less massive. So while large cluster mass certainly makes the appearance of EHB stars more likely, another factor is influencing their relative abundance. This factor is probably not past cluster mass - NGC 2419 is unlikely to have lost as much mass in its history as clusters like $\omega$ Cen and M54 because its orbit keeps it far out in the Galactic halo, minimizing the effects of Galactic tides and disk shocking. There is also no evidence of populations of greatly different age in NGC 2419 based on a superficial examination - NGC 1851 shows two distinct subgiant branches that could result from an age difference of about 1 Gyr (Milone et al. 2008).

For the clusters above, there is a hint that the fraction of EHB stars anticorrelates with metallicity. M15 is about 0.1 dex more metal poor than NGC 2419, but also by far the least massive of these clusters. $\omega$ Cen has a large metallicity spread $(-2.2 \lesssim[\mathrm{Fe} / \mathrm{H}] \lesssim-0.7)$, but the most common $[\mathrm{Fe} / \mathrm{H}] \sim-1.75$ (Johnson et al. 2008). Of the clusters with moderate EHB populations, M54 has $[\mathrm{Fe} / \mathrm{H}] \sim-1.6$ (Brown et al. 1999) and NGC 2808 has $[\mathrm{Fe} / \mathrm{H}] \sim-1.15$ (Carretta 2006). The remainder of the massive clusters (NGC 6388, NGC 6441, and 47 Tuc) are very metal rich $([\mathrm{Fe} / \mathrm{H}]>-0.7)$. This crude correlation may be related to well-known relation between $[\mathrm{Fe} / \mathrm{H}]$ and HB morphology - more metal-poor clusters tend to have bluer HB stars.

Even if we compare NGC 2419 to its nearest rival in producing EHB stars ( $\omega$ Cen), we find a lack of a clear lead. $\omega$ Cen has an HB morphology similar to NGC 2419 , though $\omega$ Cen has diverted a greater proportion of stars into the blue HB tail between the two peaks, which would imply that multiple populations should overlap to a greater degree in $\omega$ Cen. $\omega$ Cen shows very clear signs of multiple populations on the giant branch and main sequence (Bedin et al. 2004), including a blue main sequence that is more metal-rich than the majority of stars, and therefore is probably also helium enriched (Piotto et al. 2005). The number ratio of chemically enriched group to the total MS star population is similar to the ratio of $\mathrm{EHB}$ stars to the total $\mathrm{HB}$ population, and thus Piotto et al. suggested that the EHB stars are the descendants of the enriched population. In NGC 2419 , there is no evidence to date of multiple populations among red giants or main sequence stars. Although there is a little evidence consistent with slight helium enrichment in the cluster (based on post-HB evolution and the $R$ population ratio), there is no evidence that it is as strong as in $\omega$ Cen. It is potentially possible to hide multiple populations on the main sequence by increasing metallicity and helium abundance in a way to cancel out their opposing color shifts. If this is happening in NGC 2419, it would imply a smaller helium enrichment $(\Delta Y \sim 0.03)$ than is implied for $\omega$ Cen $(\Delta Y=0.14)$. Such a small helium enrichment would be consistent with the indicators presented in this paper, but would be unable to explain the two HB star populations. Spectroscopic observations of cluster giants would still be needed to determine whether a metallicity spread is truly present in the cluster.

In one sense NGC 2419 is like $\omega$ Cen: neither appears to have undergone strong dynamical relaxation (Dalessandro et al. 2008; Ferraro et al. 2006). There is little or no evidence of a correlation with concentration $c$ (McLaughlin \& van der Marel 2005) though. The three massive clusters with the largest EHB fractions (NGC 2419, $\omega$ Cen, and M54) are also largest in $R_{h}$, although 47 Tuc has similar structural properties to M54 and has no EHB stars.

At this point, we must confess that while NGC 2419 
seems to have less complicated stellar populations overall than clusters like $\omega$ Cen and NGC 2808, its relative simplicity helps to stymie attempts to draw out a coherent picture of the source of multiple populations and extreme horizontal branch stars.
We thank the anonymous referee for a careful reading of the manuscript, and gratefully acknowledge financial support from the National Science Foundation under grant AST 0507785 to E.L.S. and Michael Bolte.

\section{REFERENCES}

Bedin, L. R., Piotto, G., Anderson, J., Cassisi, S., King, I. R., Momany, Y., \& Carraro, G. 2004, ApJ, 605, L125

Boyer, M. L., McDonald, I., van Loon, J. T., Woodward, C. E., Gehrz, R. D., Evans, A., \& Dupree, A. K. 2008, AJ, 135, 1395

Brown, T. M., Smith, E., Ferguson, H. C., Sweigart, A. V., Kimble, R. A., \& Bowers, C. W. 2008, ApJ, accepted

Brown, J. A., Wallerstein, G., \& Gonzalez, G. 1999, AJ, 118, 1245

Brown, T. M., Sweigart, A. V., Lanz, T., Landsman, W. B., \& Hubeny, I. 2001, ApJ, 562, 368

Busso, G., et al. 2007, A\&A, 474, 105

Cacciari, C., Corwin, T. M., \& Carney, B. W. 2005, AJ, 129, 267

Caloi, V., \& D'Antona, F. 2005, A\&A, 435, 987

Caloi, V., \& D'Antona, F. 2007, A\&A, 463, 949

Caloi, V., \& D'Antona, F. 2008, ApJ, 673, 847

Carretta, E. 2006, AJ, 131, 1766

Carretta, E., et al. 2007, A\&A, 464, 939

Cassisi, S., Castellani, M., Caputo, F., \& Castellani, V. 2004, A\&A, 426, 641

Cassisi, S., Salaris, M., \& Irwin, A. W. 2003, ApJ, 588, 862

Castellani, V., Iannicola, G., Bono, G., Zoccali, M., Cassisi, S., \& Buonanno, R. 2006, A\&A, 446, 569

Castellani, V., et al. 2007, ApJ, 663, 1021

Catelan, M., Borissova, J., Sweigart, A. V., \& Spassova, N. 1998, ApJ, 494, 265

D’Antona, F., Bellazzini, M., Caloi, V., Fusi Pecci, F., Galleti, S., \& Rood, R. T. 2005, ApJ, 631, 868

Dalessandro, E., Lanzoni, B., Ferraro, F. R., Rood, R. T., Milone, A., Piotto, G., \& Valenti, E. 2008, ApJ, 677, 1069

Dalessandro, E., Lanzoni, B., Ferraro, F. R., Vespe, F., Bellazzini, M., \& Rood, R. T. 2008, ApJ, 681, 311

D'Cruz, N. L., Dorman, B., Rood, R. T., \& O'Connell, R. W. 1996, ApJ, 466, 359

Dolphin, A. E. 2000, PASP, 112, 1383

Dotter, A., Chaboyer, B., Jevremović, D., Baron, E., Ferguson, J. W., Sarajedini, A., \& Anderson, J. 2007, AJ, 134, 376

Ferraro, F. R., Messineo, M., Fusi Pecci, F., de Palo, M. A., Straniero, O., Chieffi, A., \& Limongi, M. 1999, AJ, 118, 1738

Ferraro, F. R., Paltrinieri, B., Pecci, F. F., Rood, R. T., \& Dorman, B. 1998, ApJ, 500, 311

Ferraro, F. R., Paltrinieri, B., Fusi Pecci, F., Rood, R. T., \& Dorman, B. 1997, MNRAS, 292, L45

Ferraro, F. R., Sollima, A., Rood, R. T., Origlia, L., Pancino, E., \& Bellazzini, M. 2006, ApJ, 638, 433

Gallart, C., Zoccali, M., \& Aparicio, A. 2005, ARA\&A, 43, 387

Grundahl, F., Catelan, M., Landsman, W. B., Stetson, P. B., \& Andersen, M. I. 1999, ApJ, 524, 242

Haft, M., Raffelt, G., \& Weiss, A. 1994, ApJ, 425, 222

Harris, W. E. 1996, AJ, 112, 1487
Harris, W. E. et al. 1997, AJ, 114, 1030

Holtzman, J. A., Burrows, C. J., Casertano, S., Hester, J. J., Trauger, J. T., Watson, A. M., \& Worthey, G. 1995, PASP, 107,1065

Iben, I. J., \& Rood, R. T. 1970, ApJ, 161, 587

Itoh, N., Hayashi, H., Nishikawa, A., \& Kohyama, Y. 1996, ApJS, 102,411

Johnson, C. I., Pilachowski, C. A., Simmerer, J., \& Schwenk, D. 2008, ApJ, 681, 1505

McLaughlin, D. E., \& van der Marel, R. P. 2005, ApJS, 161, 304

Milone, A. P., et al. 2008, ApJ, 673, 241

Moehler, S., Heber, U., \& Durell, P. R. 1997, A\&A, 317, L83

Moehler, S., Sweigart, A. V., Landsman, W. B., Hammer, N. J., \& Dreizler, S. 2004, A\&A, 415, 313

Newsham, G., \& Terndrup, D. M. 2007, ApJ, 664, 332

Origlia, L., Rood, R. T., Fabbri, S., Ferraro, F. R., Fusi Pecci, F., \& Rich, R. M. 2007, ApJ, 667, L85

Pinto, G., \& Rosino, L. 1977, A\&AS, 28, 427

Piotto, G., et al. 2002, A\&A, 391, 945

Piotto, G., et al. 2005, ApJ, 621, 777

Piotto, G., et al. 2007, ApJ, 661, L53

Recio-Blanco, A., et al. 2005, A\&A, 432, 851

Ripepi, V. et al. 2007, AJ, 667, L61

Rosenberg, A., Recio-Blanco, A., \& García-Marín, M. 2004, ApJ, 603,135

Salaris, M., Riello, M., Cassisi, S., \& Piotto, G. 2004, A\&A, 420, 911

Sandquist, E. L., \& Bolte, M. 2004, ApJ, 611, 323

Sandquist, E. L., \& Martel, A. R. 2007, ApJ, 654, L65

Sarajedini, A., et al. 2007, AJ, 133, 1658

Siegel, M. H., et al. 2007, ApJ, 667, L57

Sirianni, M., et al. 2005, PASP, 117, 1049

Stetson, P. B. 1996, PASP, 108, 851

Stetson, P. B. 2005, PASP, 117, 563

Strom, S. E., Strom, K. M., Rood, R. T., \& Iben, I., Jr. 1970, A\&A, 8, 243

Suntzeff, N. B, Kraft, R. P., \& Kinman, T. D. 1988, AJ, 95, 91

Sweigart, A. V. 1987, ApJS, 65, 95

VandenBerg, D. A., Bergbusch, P. A., \& Dowler, P. D. 2006, ApJS, 162, 375

Walker, A. R. 1994, AJ, 108, 555

Zinn, R. J., Newell, E. B., \& Gibson, J. B. 1972, A\&A, 18, 390

Zinn, R. 1974, ApJ, 193, 593

Zoccali, M., Cassisi, S., Bono, G., Piotto, G., Rich, R. M., \& Djorgovski, S. G. 2000, ApJ, 538, 289 
TABLE 4

Evolved StaRs IN NGC 2419

\begin{tabular}{|c|c|c|c|c|c|c|c|c|c|c|c|c|c|}
\hline ID & $\begin{array}{l}\delta \text { R.A. } \\
(\operatorname{arcsec})\end{array}$ & $\begin{array}{c}\delta \mathrm{DEC} \\
(\operatorname{arcsec})\end{array}$ & $\begin{array}{c}\text { F300W } \\
\text { (mag) }\end{array}$ & $\begin{array}{c}\text { F555W } \\
\text { (mag) }\end{array}$ & $\begin{array}{c}\text { F814W } \\
\text { (mag) }\end{array}$ & $\begin{array}{c}\text { F250W } \\
\text { (mag) }\end{array}$ & $\begin{array}{c}\text { F336W } \\
\text { (mag) }\end{array}$ & $\begin{array}{c}\text { F380W } \\
\text { (mag) }\end{array}$ & $\begin{array}{c}\text { F435W } \\
\text { (mag) }\end{array}$ & $\begin{array}{c}\text { F439W } \\
\text { (mag) }\end{array}$ & $\begin{array}{c}\text { F450W } \\
\text { (mag) }\end{array}$ & $\begin{array}{c}\text { F675W } \\
\text { (mag) }\end{array}$ & Notes \\
\hline \multicolumn{14}{|c|}{ Post-AGB candidates } \\
\hline PA 1 & 7.800 & 16.468 & 16.876 & 17.461 & 17.371 & & & & 17.403 & & & & ZNG2 \\
\hline PA 2 & 30.141 & -28.501 & 18.026 & 16.632 & 15.707 & & 17.390 & 17.617 & & 17.397 & 17.137 & & ZNG1 \\
\hline PA 3 & 26.780 & -14.389 & 19.339 & 17.580 & 16.632 & & & & & & & & \\
\hline PA 4 & -49.254 & -100.918 & 17.556 & 15.789 & 14.797 & & 16.576 & 16.851 & 16.426 & 16.767 & & & \\
\hline PA 5 & -8.697 & -53.664 & 19.832 & 16.662 & 15.200 & & 18.842 & 18.536 & 17.791 & 17.921 & 17.593 & & \\
\hline PA 6 & 76.915 & -185.451 & & & & & & & 17.594 & & & & \\
\hline $\mathrm{PA} 7$ & -92.211 & -73.163 & & 16.563 & 15.442 & & & & 17.828 & & & & \\
\hline \multicolumn{14}{|c|}{ supra-HB stars } \\
\hline SH 1 & 32.980 & -41.293 & 18.132 & 19.545 & 19.653 & & & & 19.414 & & & & \\
\hline SH 2 & -33.302 & 13.065 & 18.755 & 19.579 & 19.600 & & & & 19.574 & & & & \\
\hline SH 3 & 10.784 & 13.315 & 19.941 & 19.209 & 18.525 & & & & 19.661 & & & & \\
\hline SH 4 & -1.433 & -21.805 & 20.141 & 19.582 & 19.374 & 20.164 & 19.961 & 19.862 & 19.716 & 19.749 & 19.763 & 19.535 & \\
\hline SH 5 & 5.825 & -5.158 & & 19.411 & 19.013 & & & & 19.727 & & & & \\
\hline SH 6 & 5.765 & -5.179 & 19.849 & 19.062 & 18.259 & & 19.450 & 19.439 & 20.146 & 19.310 & 19.217 & 18.432 & \\
\hline SH 7 & -7.038 & -23.101 & 20.605 & 19.452 & 18.748 & 21.439 & 20.046 & 20.272 & 19.803 & 20.190 & 20.077 & 19.206 & $\mathrm{~V} 2$ \\
\hline \multicolumn{14}{|c|}{ AGB manqué candidates } \\
\hline $\mathrm{AM} 1$ & -63.325 & -13.723 & & 22.200 & 22.490 & & (2) & - 1 a & 22.058 & & & & \\
\hline AM 2 & 11.255 & -1.984 & 20.762 & 22.363 & 22.590 & & & & 22.217 & & & & \\
\hline AM 3 & -20.198 & -14.353 & 20.645 & 22.326 & 22.501 & & 20.709 & 21.975 & 22.217 & 22.310 & 22.336 & 22.466 & \\
\hline AM 4 & 25.687 & -57.545 & 20.390 & 22.599 & 22.796 & & & & 22.469 & & & & \\
\hline AM 5 & 52.320 & -55.610 & 20.649 & 22.819 & 23.059 & & & & 22.619 & & & & \\
\hline AM 6 & 43.226 & -65.388 & 20.914 & 23.055 & 23.327 & & & & 22.884 & & & & \\
\hline AM 7 & 5.899 & -27.397 & & 23.163 & 23.403 & & & & 22.981 & & & & \\
\hline AM 8 & 1.987 & -3.072 & 20.954 & 23.010 & 22.860 & 20.551 & 21.101 & 22.455 & 23.433 & 22.787 & 22.601 & 22.445 & blends with BSS in WFPC2 \\
\hline AM 9 & -40.218 & -0.963 & 21.156 & 23.312 & 23.547 & & 21.271 & 22.809 & 23.126 & 23.184 & 23.130 & 23.400 & \\
\hline AM 10 & -32.261 & -16.589 & 21.187 & 23.308 & 23.502 & & 21.378 & 22.657 & 23.174 & 22.955 & 22.996 & 23.413 & \\
\hline AM 11 & 20.290 & -122.984 & & 23.441 & 23.689 & & 21.477 & 22.890 & 23.206 & 23.162 & 23.140 & & \\
\hline AM 12 & -9.226 & 1.132 & & 23.407 & 23.867 & 20.985 & & & 23.256 & & & & \\
\hline AM 13 & -8.234 & -14.431 & 21.036 & 22.554 & 22.269 & 20.734 & 21.103 & 22.515 & 22.685 & 22.594 & 22.694 & 22.165 & looks BSS in WFC, optical WFPC2 \\
\hline AM 14 & -11.461 & 2.148 & & 23.679 & 23.867 & 21.198 & & & 23.505 & & & & \\
\hline AM 15 & -2.753 & 2.081 & 21.066 & 23.448 & 23.444 & 20.644 & 21.226 & 22.892 & 24.173 & 22.822 & 22.885 & 22.662 & almost blended with BSS in WFC \\
\hline AM 16 & 3.907 & -38.774 & 20.348 & 21.752 & 21.853 & & 20.301 & 21.457 & 21.686 & 21.640 & 21.704 & 21.822 & \\
\hline AM 17 & 25.687 & -57.545 & 20.390 & 22.599 & 22.796 & & & & 22.469 & & & & \\
\hline AM 18 & -9.150 & 44.548 & 20.530 & 24.649 & 24.042 & & & & & & & & \\
\hline AM 19 & -3.935 & -13.644 & 20.971 & 23.103 & 23.193 & 20.628 & 21.059 & 23.047 & 22.996 & 23.016 & 22.948 & 23.261 & \\
\hline AM 20 & 3.324 & 6.803 & 19.497 & 19.224 & 19.077 & & & & 19.305 & & & & \\
\hline
\end{tabular}

Note. - Table 4 is available (along with photometric errors) in its entirety via the link to the machine-readable version above. PA: Post-AGB; SH: Supra-HB; AM: AGB manqué; V: RR Lyrae variable; A: AGB; H: HB; R: RGB. The table only includes stars from the union of the ACS WFC and WFPC2 fields for proposals 7628 and 7630. 